\title{
The $G_{r}(r<5)$ of $n$-Dimensional Space \\ and
}

Their Differential Invariants for $n=r=3$.

\section{DISSERTATION}

PREGENTED TO

The Faoulty of the University of Virginia,

BY

WIILEIAM BEVERLEY STONE.

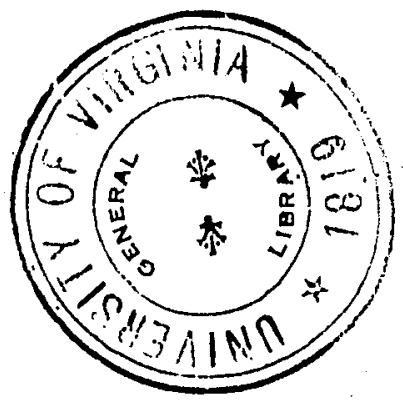

PRAEs of
THE MEW ERA PRINTING COMPAMY LAMCABtER. PA.

1908 



\section{INTRODUCTION.}

According to Prof. Sophus Lie, the "theory of Differential Equations is the most important branch of modern mathematics." During the last century, this branch of mathematical science has been developed in a number of different directions, one of the most important of which is that based on the theory of transformation groups. As is well known, this whole method was originated by Lie in 1869-70, when he showed that most of the older theories of integration owe their origin to a common source and at the same time introduced new theories of integration, based on the theory of groups.

In order to apply Lie's method to the problem of integration, it is necessary to know what group, if any, a given differential equation admits of. In his "Vorlesungen über Differentialgleichungen mit Bekannten Infinitesimalen Transformationen" and various other publications, particularly in Vol. XXXII of the Mathematische Annalen, Lie established in complete detail all differential invariants of every group in two variables, and showed how to reduce as far as possible the problem of integrating the differential equations invariant under such groups. It would seem most desirable to do the same thing, as far as possible, for groups in $x, y, z$. This great problem has been solved only for a few special cases: by Lie, for example, for the group of Euclidean movements and a few other special groups (see "Continuierliche Gruppen," Kap. 22); by Tresse for the $G_{10}$ of conform transformations (see Comptes Rendus, 1892, Tom. 114); by Dr. G. Noth in a Leipzig thesis on the differential invariants of a certain $G_{10}$. One object of the present paper is to begin the solution of this general problem in a systematic manner, by establishing the desired results for all $G_{3}$ 's in $x, y, z$.

The problem before us divides itself naturally into three parts :

I. The establishment of the normal forms of the $G_{r}(n<5)$ in $n$ variables.

II. The establishment of the differential invariants of the $G_{3}$ 's in $x, y, z:$ (i) when $y$ and $z$ are each functions of $x$; (ii) when $z$ is a function of $x$ and $y$.

III. Applications of, and remarks on, the results obtained. 


\section{CHAPTER I}

The Normax Forms of the $G_{r}(r<5)$.

In Vol. III of the "Theorie der Transformationsgruppen" by Lie and Engel, two methods of establishing these groups in $x, y, z$ are indicated; but the groups of this class - namely, those imprimitive ones which leave invariant one curve family

$$
\phi(x, y, z)=\text { const. and } \psi(x, y, z)=\text { const. }
$$

and at the same time at least one surface family

$$
\Omega(\phi, \psi)=\text { const. }
$$

- are exceedingly numerous, and, so far as the present writer is able to discover, have never been established and tabulated, as was done for the other classes. We shall make direct use of neither of the methods given by $\mathrm{Lie}$ in the reference mentioned above, but shall follow other methods frequently used by him in other connections for establishing the normal forms of groups. It is believed that the $G_{2}$ 's, given on pp. 6-7, and the tables of $G_{3}$ 's, $G_{4}$ 's, given on pp. 15-16 and pp. 29-35 of this paper are accurate and exhaustive so that they can be confidently referred to by any one desiring to use these groups.

\section{§1. Normal Forms of the $G_{2}^{\prime}$ 's of Space.}

In his "Theorie der Berührungstransformationen," Lie established in all essentials the normal forms of the $G_{2}$ 's in $x, y, z$. In deducing the $G_{2}$ 's of $n$-dimensional space, we shall follow the method used by him in the above mentioned case, and at the same time show that the four types obtained by $\mathrm{Lie}$ are exhaustive also for $n$-dimensional space. We shall use $p_{i}$ to represent $\partial f / \partial x_{i}$. .

If

$$
U_{k} f=\sum_{i=1}^{n} \xi_{k_{1}}\left(x_{1}, \cdots, x_{n}\right) p_{i} \quad(k=1,2)
$$

are two representative transformations of the group, it is well known 
THE $G_{r}(r<5)$ OF $n$-DIMENgIONAL SPACE.

that they can be assumed to satisfy one of the structures (Zusammensetzungen)

Hence

$$
\left(U_{1} U_{2}\right)=0, \quad\left(U_{1} U_{2}\right)=U_{1} \text {. }
$$

$$
U_{1} f=0, \quad U_{2} f=0
$$

will in each case define a complete system of at most two members in the $n$-variables $x_{1}, \ldots, x_{n}$. Hence this complete system will have at least $n-2$ common solutions, which are independent functions of the variables $x_{1}, \ldots, x_{n}$. If $\psi_{3}\left(x_{1}, \ldots, x_{n}\right), \ldots, \psi_{n}\left(x_{1}, \ldots, x_{n}\right)$ are the solutions of the complete system, we may without loss of generality assume the $\psi_{i}$ to be independent functions with regard to the $n-2$ variables $x_{3}, \cdots, x_{n}$; hence we may introduce as a new system of independent variables the $\bar{x}_{j}$ defined by

$$
\bar{x}_{1}=x_{1}, \quad \bar{x}_{2}=x_{2}, \quad \bar{x}_{1}=\psi_{0}\left(x_{1}, \cdots, x_{n}\right) \quad(s=3, \cdots, n) .
$$

In these new variables, we have

$$
\bar{U}_{k} f=\bar{\xi}_{k, 1}\left(\bar{x}_{1}, \ldots, \bar{x}_{n}\right) \bar{p}_{1}+\bar{\xi}_{k, 2}\left(\bar{x}_{1}, \cdots, \bar{x}_{n}\right) \bar{p}_{2} \quad(k=1,2),
$$

where the $\bar{x}_{3}, \ldots, \bar{x}_{n}$ play the rôle of constants. One of these transformations $\bar{U}_{1} f$ (say) can, by the introduction of new variables

$\bar{x}_{1}=\phi_{1}\left(\bar{x}_{1}, \ldots, \bar{x}_{n}\right), \quad \bar{x}_{2}=\phi_{2}\left(\bar{x}_{1}, \ldots, \bar{x}_{n}\right), \quad \bar{x}_{3}=\bar{x}_{3}, \ldots, \bar{x}_{n}=\bar{x}_{n}$,

always be thrown into the form of a translation $\bar{p}_{1}$, or let us say simply $p_{1}$. Suppose that by the introduction of the new variables the other transformation becomes

$$
U_{2} f=\xi_{1}\left(x_{1}, \cdots, x_{n}\right) p_{1}+\xi_{2}\left(x_{1}, \cdots, x_{n}\right) p_{2} .
$$

Now there are two cases according as the two transformations have the same path-curves or not. We consider these in order.

I. When the path-curves are the same,

$$
U_{2} f=\rho\left(x_{1}, \cdots, x_{n}\right) p_{1} .
$$

$$
\left(U_{1} U_{2}\right)=0: \quad \therefore\left(p_{1}, \rho\left(x_{1}, \cdots, x_{n}\right) p_{1}\right) \equiv \rho_{x_{1}} \cdot p_{l} l=0,
$$

Hence $\rho$ is free of $x_{1}$. Also $\rho$ cannot be a constant, since then there would be only one transformation. As $\rho$ must contain at least one 
WILLIAM B. STONE.

of the variables $x_{2}, \cdots, x_{n}$, suppose that it contains $x_{j}$. Then we may introduce $\rho$ as a new $\bar{x}_{j}$, so that

$$
\bar{U}_{2} f \equiv \bar{x}_{j} p_{1}
$$

Hence for this case we have the group

$$
p_{1}, x_{j} p_{1} \quad(j>1) \text {. }
$$

(ii) $\left(U_{2}, U_{2}\right)=U_{1}: \quad \therefore\left(p_{1}, \rho\left(x_{1}, \ldots, x_{n}\right) p_{1}\right) \equiv \rho_{x_{1}} \cdot p_{1}=p_{1}$.

Hence $\rho \equiv x_{1}+\alpha\left(x_{2}, \cdots, x_{n}\right)$.

Putting $\bar{x}_{1}=x_{1}+\alpha\left(x_{2}, \cdots, x_{n}\right)$, we have $\bar{U}_{2} f=\bar{x}_{1} \bar{p}_{1}$, while $U_{1} f \equiv \bar{p}_{1}$. Hence for this case we have the group

$$
p_{1}, x_{1} p_{1} \text {. }
$$

II. When the path-curves of the transformations are different,

$$
U_{2} f \neq \rho\left(x_{1}, \cdots, x_{n}\right) U_{1} f .
$$

For this case $U_{1} f \equiv p_{1}, U_{2} f \equiv \xi_{1}\left(x_{1}, \cdots, x_{n}\right) p_{1}+\xi_{2}\left(x_{1}, \cdots, x_{n}\right) p_{2^{\prime}}$

(i) $\left(U_{1} U_{2}\right)=0: \quad \therefore\left(p_{1}, \xi_{1} p_{1}+\xi_{2} p_{2}\right) \equiv \xi_{x_{1}} p_{1}+\xi_{x_{1}} p_{2}=0$.

Hence $\xi_{1}$ and $\xi_{2}$ are free of $x_{1}$, so that

$$
U_{2} f \equiv \alpha\left(x_{2}, \cdots, x_{n}\right) p_{1}+\beta\left(x_{2}, \cdots, x_{n}\right) p_{2} \quad(\beta \neq 0) .
$$

Introducing as new variables

we have

$$
\bar{x}_{1}=x_{1}-\int \frac{\alpha}{\beta} d x_{2}, \quad \bar{x}_{2}=\int \frac{d x_{2}}{\beta},
$$

$$
\bar{U}_{1} f=\bar{p}_{1}, \quad \bar{U}_{2} f=\bar{p}_{2} .
$$

Hence for this case, we obtain the group

$$
p_{1}, p_{2} \text {. }
$$

(ii) $\left(U_{1} U_{2}\right)=U_{1}: \quad \therefore\left(p_{1}, \xi_{1} p+\xi_{2} p_{2}\right) \equiv \xi_{x_{1}} p_{1}+\xi_{x_{1}} p_{2}=p_{1}$.

Hence

$$
\xi_{1} \equiv x_{1}+\alpha\left(x_{2}, \cdots, x_{n}\right), \quad \xi_{2} \equiv \beta\left(x_{2}, \cdots, x_{n}\right) \quad(\beta \neq 0) .
$$


THE $G_{r}(r<5)$ OF $n$-DIMENGIONAL SPACE.

Introducing as new variables

$$
\bar{x}_{1}=x_{1}+\phi\left(x_{2}, \cdots, x_{n}\right), \quad \bar{x}_{2}=\epsilon^{\int \frac{d x_{2}}{\beta}},
$$

and choosing $\phi$ so as to make the arbitrary function in $\xi_{1}$ disappear, we obtain

$$
\bar{U}_{1} f=\bar{p}_{1}, \quad \bar{U}_{2} f=\bar{x}_{1} \bar{p}_{1}+\bar{x}_{2} \bar{p}_{2} .
$$

Without changing the first transformation, the second can be thrown into the symmetrical form

$$
U_{2} f \equiv \sum_{i=1}^{n} x_{i} p_{i}
$$

by introducing $x_{1}=\bar{x}_{1}, x_{2}=\bar{x}_{2}, x_{j}=\bar{x}_{2} \bar{x}_{j}(j=3, \ldots, n)$. Hence for this case we obtain the group

$$
p_{1}, \sum_{i=1}^{n} x_{i} p_{i} \text {. }
$$

As all possible cases have been considered these four types must be exhaustive.

\$2. Normal Forms of the $G_{3}$ 's of Space.

Lie has shown that' the representative transformations $U_{1} f, U_{2} f$, $U_{3} f$, of a $G_{3}$ in $n$ variables can always be chosen so that the $G_{3}$ has one of the structures (Zusammensetzungen) $I, \ldots, V$, below (see "Theorie der Transformationsgruppen," Dritter Abschnitt, Kap. 28). Under each one of these structures we must consider the various cases which arise when the transformations satisfy two independent linear relations, one such relation, or no such relation. These will be taken up in order.

I. Let the transformations satisfy the structiure

$$
\left(U_{1} U_{2}\right) \equiv U_{1}, \quad\left(U_{1} U_{3}\right) \equiv 2 U_{2}, \quad\left(U_{2} U_{3}\right) \equiv U_{3} .
$$

(1) Suppose there are two independent linear relations connecting the transformations, and let them be

$$
\rho_{1} U_{1}+\rho_{2} U_{2}+\rho_{3} U_{3}=0, \quad \sigma_{1} U_{1}+\sigma_{2} U_{2}+\sigma_{3} U_{3}=0,
$$

$\rho_{i}$ and $\sigma_{i}$ being functions of $x_{1}, \ldots, x_{n}$.

Solving these two equations for $U_{2}$ and $U_{3}$, we obtain

$$
U_{2} f \equiv \rho\left(x_{1}, \ldots, x_{n}\right) U_{1} f, \quad U_{3} f \equiv \sigma\left(x_{1}, \ldots, x_{n}\right) U_{1} f .
$$


Hence if we suppose, as can be done, that $U_{1} f$ has been thrown into the form of a translation $p_{1}$, we have

$$
U_{2} f \equiv \rho\left(x_{1}, \cdots, x_{n}\right) p_{1}, \quad U_{3} f \equiv \sigma\left(x_{1}, \cdots, x_{n}\right) p_{1} .
$$

Since the path curves of $U_{1}$ and $U_{2}$, which form a $G_{2}$, are the same, we can, by the preceding section, put

$$
U_{2} f \equiv x_{1} p_{1} \text {. }
$$

Substituting in the structure, we find

whence

$$
\begin{aligned}
& \left(U_{1} U_{3}\right) \equiv\left(p_{1}, \sigma p_{1}\right) \equiv \sigma_{x_{1}} p_{1}=2 x_{1} p_{1}, \quad \text { or } \sigma_{x_{1}}=2 x_{1} \\
& \left(U_{2} U_{3}\right) \equiv\left(x_{1} p_{1}, \sigma p_{1}\right) \equiv 2 x_{1}^{2} p_{1}-\sigma p_{1}=\sigma p_{1},
\end{aligned}
$$

$$
\sigma=x_{1}^{2} \text { and } U_{3} f \equiv x_{1}^{2} p_{1} .
$$

Hence for this case we have the group

$$
p_{1}, x_{1} p_{1}, x_{1}^{2} p_{1} \text {. }
$$

(2) Suppose there is only one linear relation connecting the transformations, namely

$$
\rho_{1} U_{1}+\rho_{2} U_{2}+\rho_{3} U_{3}=0,
$$

$\rho_{i}$ being functions of $x_{1}, \cdots, x_{n}$. Since the $U_{i} f$ do not occur symmetrically in the structure of the $G_{3}$, we shall further subdivide this case according as :

$$
\begin{array}{ll}
\rho_{1} \equiv 0, \rho_{2} \cdot \rho_{3} \neq 0 ; & \text { (ii) } \rho_{2} \equiv 0, \rho_{1} \cdot \rho_{3} \neq 0 ; \\
\rho_{3} \equiv 0, \rho_{1} \cdot \rho_{2} \neq 0 ; & \text { (iv) } \rho_{1} \cdot \rho_{2} \cdot \rho_{3} \neq 0 .
\end{array}
$$

$$
\rho_{1} \equiv 0, \quad \rho_{2} \cdot \rho_{3} \neq 0 \text {. }
$$

Then $U_{3} f \equiv \rho\left(x_{1}, \cdots, x_{n}\right) U_{2} f$, while $U_{1} f$ and $U_{2} f$ are not connected by any linear relation, i. $\theta_{\text {., }}$

$$
U_{2} f \neq \sigma\left(x_{1}, \cdots, x_{n}\right) U_{1} f .
$$

If $U_{1} f \equiv p_{1}$, we must have by the preceding section

$$
U_{2} f \equiv x_{1} p_{1}+x_{2} p_{2} \text {. }
$$

Hence by the structure, we obtain

$$
\left(p_{1}, \rho\left(x_{1} p_{1}+x_{2} p_{2}\right)\right) \equiv\left(\rho+x_{1} \rho_{x_{1}}\right) p_{1}+x_{2} \rho_{x_{1}} p_{2} \equiv 2\left(x_{1} p_{1}+x_{2} p_{2}\right) \text {. }
$$


From this relation

$$
\rho+x_{1} \rho_{x_{1}}=2 x_{1}, \quad x_{2} \rho_{x_{1}}=2 x_{2},
$$

i. e., $\rho=0$, consequently there is no $G_{3}$ for this case. In the same way it is shown that the subdivisions (ii) and (iii) above give no groups.

Let us consider the last of these, i. e.,

$$
\rho_{1} \cdot \rho_{2} \cdot \rho_{3} \neq 0 \text {. }
$$

For this case we may take $U_{1} f=p_{1}, U_{2} f=x_{1} p_{1}+x_{2} p_{2}$, since no linear relation connects any two of the transformations. Also we can take

$$
U_{3} f \equiv \rho U_{1} f+\sigma U_{2} f \quad \rho \cdot \sigma \neq 0,
$$

$\rho$ and $\sigma$ being functions of $x_{1}, \ldots, x_{n}$.

Thus we have

$$
U_{3} f \equiv\left(\rho+x_{1} \sigma\right) p_{1}+x_{2} \sigma p_{2} .
$$

Substituting in the structure

$$
\begin{array}{r}
\left(p_{1},\left(\rho+x_{1} \sigma\right) p_{1}+x_{2} \sigma p_{2}\right) \equiv\left(\rho_{x_{1}}+x_{1} \sigma_{x_{1}}+\sigma\right) p_{1}+x_{2} \sigma_{x_{1}} p_{2} \equiv 2\left(x_{1} p_{1}+x_{2} p_{2}\right) \\
\left(x_{1} p_{1}+x_{2} p_{2},\left(\rho+x_{1} \sigma\right) p_{1}+x_{2} \sigma p_{2}\right) \equiv 2 x_{1}\left(x_{1} p_{1}+x_{2} p_{2}\right) \\
+x_{2}\left\{\left(\rho_{x_{2}}+x_{1} \sigma_{x_{2}}\right) p_{1}+\left(x_{2} \sigma_{x_{2}}+\sigma\right) p_{2}\right\}-\left(\rho+x_{1} \sigma\right) p_{1}-x_{2} \sigma p_{2} \\
=\left(\rho+x_{1} \sigma\right) p_{1}+x_{2} \sigma p_{2} .
\end{array}
$$

Equating coefficients in these two relations, we have

$$
\begin{aligned}
& \rho_{x_{1}}+x_{1} \sigma_{x_{1}}+\sigma=2 x_{1}, \quad 2 x_{1} x_{2}+x_{2}^{2} \sigma_{x_{2}}=x_{2} \sigma, \\
& 2 x_{1}^{2}+x_{2} \rho_{x_{2}}+x_{1} x_{2} \sigma_{x_{2}}=2 \rho+2 x_{1} \sigma, \quad . \quad x_{2} \sigma_{x_{1}}=2 x_{2} \text {. }
\end{aligned}
$$

From these relations we obtain immediately

$$
\sigma \equiv 2 x_{1}+x_{2} \cdot \lambda, \quad \rho \equiv-x_{1}^{2}-x_{1} x_{2} \lambda+x_{2}^{2} \mu,
$$

$\lambda$ and $\mu$ being functions of $x_{3}, \cdots, x_{n}$.

Hence

$$
U_{3} f \equiv\left(x_{1}^{2}+x_{2}^{2} \mu\right) p_{1}+\left(2 x_{1} x_{2}+x_{2}^{2} \lambda\right) p_{2} .
$$

Introducing as new $x_{1}, \bar{x}_{1}=x_{1}+x_{2} \phi\left(x_{3}, \cdots, x_{n}\right)$, and choosing $\phi$ so that $\phi^{2}-\phi \lambda-\mu=0$, we obtain 


$$
\begin{gathered}
\bar{U}_{3} f \equiv \bar{x}_{1}^{2} \bar{p}_{1}+\left[2 \bar{x}_{1} x_{2}+x_{2}^{2} \lambda_{1}\left(x_{3}, \cdots, x_{n}\right)\right] p_{2}, \\
\lambda_{1}\left(x_{3}, \cdots, x_{n}\right) \equiv \lambda\left(x_{3}, \cdots, x_{n}\right)-2 \phi\left(x_{3}, \ldots, x_{n}\right) .
\end{gathered}
$$

Now if $\lambda_{1}=0$, then $U_{3} f \equiv x_{1}^{2} p_{1}+2 x_{1} x_{2} p_{2}$, and we have the $G_{3}$

$$
p_{1}, x_{1} p_{1}+x_{2} p_{2}, x_{1}^{2} p_{1}+2 x_{1} x_{2} p_{2} \text {. }
$$

If $\lambda_{1} \neq 0$, put $\bar{x}_{2}=x_{2} \lambda_{1}$, and we have the $G_{3}$

$$
p_{1}, x_{1} p_{1}+x_{2} p_{2}, x_{1}^{2} p_{1}+\left(2 x_{1} x_{2}+x_{2}^{2}\right) p_{2} \text {. }
$$

(8) Suppose there is no linear relation connecting the transformations. Then we may take

$$
U_{1} f \equiv p_{1}, \quad U_{2} f \equiv x_{1} p_{1}+x_{2} p_{2},
$$

and as $U_{i} f=0(i=1,2,3)$ form a complete system of three members in $n$-variables, we may assume the variables so chosen that $U_{3} f$ has the form

$$
U_{3} f \equiv \xi_{1}\left(x_{1}, \ldots, x_{n}\right) p_{1}+\xi_{2}\left(x_{1}, \ldots, x_{n}\right) p_{2}+\xi_{3}\left(x_{1}, \ldots, x_{n}\right) p_{3} .
$$

Substituting in the structure

$$
\left(U_{1} U_{3}\right) \equiv\left(p_{1}, \xi_{1} p_{1}+\xi_{2} p_{2}+\xi_{3} p_{3}\right) \equiv \xi_{x_{1}} p_{1}+\xi_{x_{1}} p_{2}+\xi_{x_{1}} p_{3}
$$

Whence

$$
=2\left(x_{1} p_{1}+x_{2} p_{2}\right) \text {. }
$$

$$
\xi_{1} \equiv x_{1}^{2}+\alpha\left(x_{2}, \cdots, x_{n}\right), \quad \xi_{2} \equiv 2 x_{1} x_{2}+\beta\left(x_{2}, \cdots, x_{n}\right),
$$

So that

$$
\xi_{3} \equiv \gamma\left(x_{2}, \cdots, x_{n}\right) \quad(\gamma \neq 0) .
$$

$$
U_{3} f \equiv\left(x_{1}^{2}+\alpha\right) p_{1}+\left(2 x_{1} x_{2}+\beta\right) p_{2}+\gamma \cdot p_{3} \text {. }
$$

Next we have

$$
\begin{aligned}
\left(U_{2} U_{3}\right) \equiv & x_{1}\left(2 x_{1} p_{1}+2 x_{2} p_{2}\right)+x_{2}\left\{\alpha_{x_{2}} p_{1}+\left(2 x_{1}+\beta_{x_{2}}\right) p_{2}+\gamma_{x_{3}} p_{3}\right\} \\
& -\left(x_{1}^{2}+\alpha\right) p_{1}-\left(2 x_{1} x_{2}+\beta\right) p_{2}=\left(x_{1}^{2}+\alpha\right) p_{1}+\left(2 x_{1} x_{2}+\beta\right) p_{2}+\gamma p_{3} .
\end{aligned}
$$

From this relation

$$
\begin{gathered}
2 x_{1}^{2}+x_{2} \cdot \alpha_{x_{2}}=2 x_{1}^{2}+2 \alpha \\
2 x_{1} x_{2}+2 x_{1} x_{2}+x_{2} \beta_{x_{8}}=4 x_{1} x_{2}+2 \beta, \\
x_{2} \gamma_{x_{2}}=\gamma,
\end{gathered}
$$


so that

$\alpha \equiv x_{2}^{2} \lambda\left(x_{3}, \cdots, x_{n}\right), \beta \equiv x_{2}^{2} \mu\left(x_{3}, \ldots, x_{n}\right), \gamma \equiv x_{2} \nu\left(x_{3}, \cdots, x_{n}\right)$, and

$$
U_{3} f \equiv\left(x_{1}^{2}+x_{2}^{2} \lambda\right) p_{1}+\left(2 x_{1} x_{2}+x_{2}^{2} \mu\right) p_{2}+x_{2} \nu p_{3} \quad(\nu \neq 0) .
$$

Introduce $\bar{x}_{3}=\int d x_{3} / \nu$ and denote the new arbitrary functions in the coefficients of $p_{1}, p_{2}$, by $\lambda_{1}, \mu_{1}$, respectively. Then

$$
\bar{U}_{3} f \equiv\left(x_{1}^{2}+x_{2}^{2} \lambda_{1}\right) p_{1}+\left(2 x_{1} x_{2}+x_{2}^{2} \mu_{1}\right) p_{2}+x_{2} \bar{p}_{3} .
$$

Introduce again

$$
\bar{x}_{1}=x_{1}+x_{2} \phi\left(x_{3}, \cdots, x_{n}\right),
$$

and choose $\phi$ to satisfy

Then

$$
\lambda_{1}+\mu_{1} \phi-\phi^{2}+\phi_{x_{3}}=0 .
$$

$$
U_{3} f \equiv \bar{x}_{1}^{2} \bar{p}_{1}+\left[2 \bar{x}_{1} x_{2}+x_{2}^{2} \rho\left(x_{3}, \cdots, x_{n}\right)\right] p_{2}+x_{2} p_{3},
$$

where, for brevity, we substitute

Now introduce

$$
\rho\left(x_{3}, \cdots, x_{n}\right) \equiv \mu_{1}-2 \phi \text {. }
$$

$$
\bar{x}_{2}=x_{2} \psi\left(x_{3}, \cdots, x_{n}\right), \quad \bar{x}_{3}=\int \psi d x_{3},
$$

in which $\psi$ must be different from zero. Leaving off the bars, we have finally

and thus the $G_{3}$

$$
U_{3} f \equiv x_{1}^{2} p_{1}+2 x_{1} x_{2} p_{2}+x_{2} p_{3}
$$

$$
p_{1}, x_{1} p_{1}+x_{2} p_{2}, x_{1}^{2} p_{1}+2 x_{1} x_{2} p_{2}+x_{2} p_{3} \text {. }
$$

II. Let the three transformations satisfy the structure

$$
\left(U_{1} U_{2}\right)=0, \quad\left(U_{1} U_{3}\right)=U_{1}, \quad\left(U_{2} U_{3}\right)=c U_{2}
$$

(1) If two linear independent relations exist among the transformations, then as in the preceding case we have

$$
U_{2} f \equiv \rho\left(x_{1}, \cdots, x_{n}\right) p_{1}, \quad U_{3} f \equiv \sigma\left(x_{1}, \cdots, x_{n}\right) p_{1},
$$

if, as usual, we assume that $U_{1} f \equiv p_{1}$. 
Since the path curves for $U_{1} f$ and $U_{2} f$ are the same, we must by the preceding section take

Substituting in the structure

$$
U_{2} f=x_{k} p_{1}
$$

$$
\left(p_{1}, \sigma p_{1}\right) \equiv \sigma_{x_{1}} p_{1} \equiv p_{1}, \quad\left(x_{k} p_{1}, \sigma p_{1}\right) \equiv x_{k} p_{1} \equiv c x_{k} p_{1} .
$$

Hence for this case to exist $c=1$ and

$$
\sigma \equiv x_{1}+\alpha\left(x_{2}, \cdots, x_{n}\right) .
$$

Introducing $\bar{x}_{1}=x_{1}+\alpha$, we have $U_{3} f=x_{1} p_{1}$. Hence the $G_{3}$

$$
p_{1}, x_{k s} p_{1}, x_{1} p_{1} \quad(k>1) \text {. }
$$

(2) If one linear relation exists, we have four cases as under I, page 8.

so that

$$
\rho_{1}=0, \quad \rho_{2} \cdot \rho_{3} \neq 0,
$$

$$
U_{3} f=\rho\left(x_{1}, \ldots, x_{n}\right) U_{2} f, \quad U_{2} f \neq \sigma\left(x_{1}, \ldots, x_{n}\right) U_{1} f .
$$

Hence for $\left(U_{1} U_{2}\right)=0$, we must by the preceding section have

$$
U_{1} f \equiv p_{1}, \quad U_{2} f \equiv p_{2}, \quad U_{3} f \equiv \rho p_{2} .
$$

Evidently this case gives a contradiction under the above structure, so that there is no $G_{3}$ for it.

(ii)

In this case

$$
\rho_{2}=0, \quad \rho_{1} \cdot \rho_{3} \neq 0 .
$$

$$
U_{3} f \equiv \rho\left(x_{1}, \cdots, x_{n}\right) U_{1} f, \quad U_{2} f \neq \sigma\left(x_{1}, \cdots, x_{n}\right) U_{1} f .
$$

Hence by the preceding section we must take

$$
U_{1} f \equiv p_{1}, \quad U_{2} f \equiv p_{2}, \quad U_{3} f \equiv \rho\left(x_{1}, \ldots, x_{n}\right) p_{1} .
$$

Applying the structure we see that this case holds only when $c=0$. Then $\rho_{x_{1}}=1, \rho_{x_{2}}=0$. Whence $\rho \equiv x_{1}+\alpha\left(x_{3}, \cdots, x_{n}\right)$. Putting $x_{1} \equiv x_{1}+\alpha$, we have $U_{3} f \equiv \bar{x}_{1} p_{1}$ and thus the $G_{3}$

$$
\begin{gathered}
p_{1}, p_{2}, x_{1} p_{1} . \\
\rho_{3}=0, \quad \rho_{1} \cdot \rho_{2} \neq 0 .
\end{gathered}
$$


THE $G_{r}(r<5)$ OF $n$-DIMENSIONAL SPACE,

Hence $U_{2} f \equiv \rho\left(x_{1}, \cdots, x_{n}\right) U_{1} f$, and by the preceding section, we have

$$
U_{1} f \equiv p_{1}, \quad U_{2} f \equiv x_{2} p_{1} .
$$

It remains to determine $U_{3} f$, for which there will be two cases and which according to hypothesis is not linearly connected with $U_{1} f$ and $U_{2} f$. Now $U_{i} f=0(i=1,2,3)$ form a complete system, of which, since two of the equations are independent, there are $n-2$ independent solutions. Now we make two cases according as $\xi_{2}$ in

$(a)$ is not zero; or $(b)$ is zero.

$$
U_{3} f \equiv \sum_{i=1}^{n} \xi_{i} p_{i}
$$

$$
\xi_{2}\left(x_{1}, \cdots, x_{n}\right) \neq 0 \text {. }
$$

$x_{1}$ and $x_{2}$ are not solutions of the complete system and can therefore be introduced as new variables. Introducing also $\bar{x}_{j}=\psi_{j}\left(x_{1}, \ldots, x_{n}\right)$, $(j=3, \cdots, n)$ where the $\psi_{j}$ are the $n-2$ independent solutions, we have

$$
\bar{U}_{3} f=\bar{\xi}_{1}\left(\bar{x}_{1}, \ldots, \bar{x}_{n}\right) \bar{p}_{1}+\bar{\xi}_{2}\left(\bar{x}_{1}, \ldots, \bar{x}_{n}\right) \bar{p}_{2} .
$$

Leaving off the bars and applying the structure, we have immediately $\xi_{1} \equiv x_{1}+\alpha\left(x_{2}, \cdots, x_{n}\right), \xi_{2} \equiv(1-c) x_{2}$. Introducing again $\bar{x}_{1}=x_{1}+\phi\left(x_{2}, \cdots, x_{n}\right)$ and choosing $\phi$ so that

we get

$$
\alpha-\phi+(1-c) x_{2} \phi_{x_{1}}=0 \text {, }
$$

$$
\bar{U}_{3} f \equiv \bar{x}_{1} \bar{p}+(1-c) x_{2} p_{2}
$$

Thus we have the group

$$
\frac{p_{1}, x_{2} p_{1}, x_{1} p_{1}+(1-c) x_{2} p_{2}}{\xi_{2}\left(x_{1}, \cdots, x_{n}\right)=0 .}
$$

In this case $x_{2}$ will be a solution of the system, but at least one of the variables, $x_{3}$ (say), will not be a solution. We can introduce as new variables $\bar{x}_{1}=x_{1}, \bar{x}_{3}=x_{3}$ and the $n-2$ solutions

Then

$$
\bar{x}_{2}=x_{2}, \quad \bar{x}_{j}=\psi_{j}\left(x_{1}, \cdots, x_{n}\right) \quad(j=4, \cdots, n) .
$$

$$
\bar{U}_{3} f \equiv \bar{\xi}_{1}\left(\bar{x}_{1}, \cdots, \bar{x}_{n}\right) \bar{p}_{1}+\bar{\xi}_{3}\left(\bar{x}_{1}, \cdots, \bar{x}_{n}\right) \bar{p}_{3} \text {. }
$$


Leaving off the bars, and applying the structure we find

$$
\dot{\bar{x}}_{1}=x_{1}+\alpha\left(x_{2}, \cdots, x_{n}\right), \quad \vec{x}_{3}=\beta\left(x_{2}, \cdots, x_{n}\right) .
$$

Introducing again

$$
\bar{x}_{1}=x_{1}+\phi\left(x_{2}, \cdots, x_{n}\right), \quad \bar{x}_{3}=\int \frac{d x_{3}}{\beta},
$$

and determining $\phi$ from $\alpha-\phi+\beta \cdot \phi_{x_{3}}=0$, we have

Thus we get the $G_{3}$

$$
U_{3} f \equiv x_{1} p_{1}+p_{3} \text {. }
$$

$$
\frac{p_{1}, x_{2} p_{1}, x_{1} p_{1}+p_{3}}{\rho_{1} \cdot \rho_{2} \cdot \rho_{3} \neq 0 .} .
$$

By $\S 1$, we must have in this case,

Then

$$
U_{1} f \equiv p_{1}, \quad U_{2} f \equiv p_{2} .
$$

$$
U_{3} f \equiv \rho\left(x_{1}, \cdots, x_{n}\right) p_{1}+\sigma\left(x_{1}, \cdots, x_{n}\right) p_{2} .
$$

The structure gives

whence

$$
\begin{aligned}
& \left(p_{1}, \rho p_{1}+\sigma p_{2}\right) \equiv \rho_{x_{1}} p_{1}+\sigma_{x_{1}} p_{2}=p_{1}, \\
& \left(p_{2}, \rho p_{1}+\sigma p_{2}\right) \equiv \rho_{x_{2}} p_{1}+\sigma_{x_{2}} p_{2}=c p_{2},
\end{aligned}
$$$$
\rho \equiv x_{1}+\alpha\left(x_{3}, \cdots, x_{n}\right), \quad \sigma \equiv c x_{2}+\beta\left(x_{3}, \cdots, x_{n}\right) .
$$

If $c \neq 0$, we can put

$$
\bar{x}_{1}=x_{1}+\alpha, \bar{x}_{2}=x_{2}+\frac{\beta}{c} .
$$

Then $\bar{U}_{3} f \equiv x_{1} p_{1}+c x_{2} p_{2}$ and we have the $G_{3}$

$$
p_{1}, p_{2}, x_{1} p_{1}+c x_{2} p_{2} \quad(c \neq 0) .
$$

If $c=0$, put $\bar{x}_{3} \equiv \beta\left(x_{3}, \cdots, x_{n}\right)$ and we have the $G_{3}$

$$
p_{1}, p_{2}, x_{1} p_{1}+x_{3} p_{2} \text {. }
$$

(3) Finally let us suppose that no linear relation exists among the transformations. Then by $\S 1$, we must have

$$
U_{1} f \equiv p_{1}, \quad U_{2} f \equiv p_{2} .
$$

Now, since $U_{i} f=0$ form a complete system of three independent 
THE $G_{r}(r<5)$ OF n-DIMENSIONAL SPAOE.

equations, there are $n-3$ common solutions $\psi_{1}\left(x_{1}, \ldots, x_{n}\right), \ldots$, $\psi_{n}\left(x_{1}, \cdots, x_{n}\right)$, (say).

Introducing as new variables

$$
\bar{x}_{1}=x_{1}, \quad \bar{x}_{2}=x_{2}, \quad \bar{x}_{3}=x_{3}, \quad \bar{x}_{j}=\psi_{j}\left(x_{1}, \cdots, x_{n}\right) \quad(j=4, \cdots, n),
$$

we have $\bar{U}_{3} f \equiv \xi_{1}\left(\bar{x}_{1}, \ldots, \bar{x}_{n}\right) \bar{p}_{1}+\xi_{2}\left(\bar{x}_{1}, \ldots, \bar{x}_{n}\right) \bar{p}_{2}+\xi_{3}\left(\bar{x}_{1}, \ldots, \bar{x}_{n}\right) p_{3}$. By the structure

$$
\begin{aligned}
& \left(p_{1}, \xi_{1} p_{1}+\xi_{2} p_{2}+\xi_{3} p_{3}\right) \equiv \xi_{1 x_{1}} p_{1}+\xi_{2 x_{1}} p_{2}+\xi_{3 x_{1}} p_{3}=p_{1} \\
& \left(p_{2}, \xi_{1} p_{1}+\xi_{2} p_{2}+\xi_{3} p_{3}\right) \equiv \xi_{x_{2}} p_{1}+\xi_{2_{2}} p_{2}+\xi_{3 x_{2}} p_{3}=c p_{2}
\end{aligned}
$$

whence

$\xi_{1}=x_{1}+\alpha\left(x_{3}, \cdots, x_{n}\right), \quad \xi_{2}=c x_{2}+\beta\left(x_{3}, \cdots, x_{n}\right), \quad \xi_{3}=\gamma\left(x_{3}, \cdots, x_{n}\right)$.

By hypothesis $\gamma$ cannot be zero. Introducing $\bar{x}_{1}=x_{1}+\phi\left(x_{3}, \cdots, x_{n}\right), \quad \bar{x}_{2}=x_{2}+\psi\left(x_{3}, \cdots, x_{n}\right), \quad \bar{x}_{3}=\int \frac{d x_{3}}{\gamma}$, and choosing $\phi$ and $\psi$ so as to make the functions in $\xi_{1}$ and $\xi_{2}$ disappear, we have

and thus the group

$$
\bar{U}_{3} f \equiv x_{1} p_{1}+c x_{2} p_{2}+p_{3}
$$

$$
p_{1}, p_{2}, x_{1} p_{1}+c x_{2} p_{2}+p_{3}
$$

The treatment for the remaining structures is quite similar to the preceding; we shall therefore not give the calculations in detail, but shall, in concluding this section, give the results in a complete table of all $G_{3}$ 's classified according to the various structures.

I. $\quad\left(U_{1} U_{2}\right)=U_{1}, \quad\left(U_{1} U_{3}\right)=2 U_{2}, \quad\left(U_{2} U_{3}\right)=U_{3}$.

$$
p_{1}, x_{1} p_{1}, x_{1}^{2} p_{1} \quad p_{1}, x_{1} p_{1}+x_{2} p_{2}, x_{1}^{2} p_{1}+2 x_{1} x_{2} p_{2}+x_{2} p_{3}
$$

$$
p_{1}, x_{1} p_{1}+x_{2} p_{2}, x_{1}^{2} p_{1}+2 x_{1} x_{2} p_{2}
$$$$
p_{1}, x_{1} p_{1}+x_{2} p_{2}, x_{1}^{2} p_{1}+\left(2 x_{1} x_{2}+x_{2}^{2}\right) p_{2}
$$ 
II. $\quad\left(U_{1} U_{2}\right)=0,\left(U_{1} U_{3}\right)=U_{1}, \quad\left(U_{2} U_{3}\right)=c U_{2}$.

$$
\begin{array}{|l|l|l|l|l|}
\hline p_{1}, x_{k} p_{1}, x_{1} p_{1} & p_{1}, p_{2}, x_{1} p_{1} & p_{1}, x_{2} p_{1}, x_{1} p_{1}+(1-c) x_{2} p_{2} \\
\hline p_{1}, x_{2} p_{1}, x_{1} p_{1}+p_{3} & p_{1}, p_{2}, x_{1} p_{1}+c x_{2} p_{2} & p_{1}, p_{2}, x_{1} p_{1}+x_{3} p_{2} \\
p_{1}, p_{2}, x_{1} p_{1}+c x_{2} p_{2}+p_{3}
\end{array}
$$

III. $\left(U_{1} U_{2}\right)=0,\left(U_{1} U_{3}\right)=U_{1},\left(U_{2} U_{3}\right)=U_{1}+U_{2}$.

$$
\begin{gathered}
p_{1}, x_{2} p_{1}, x_{1} p_{1}-p_{2} \quad p_{1}, p_{2},\left(x_{1}+x_{2}\right) p_{1}+x_{2} p_{2} \\
p_{1}, p_{2},\left(x_{1}+x_{2}\right) p_{1}+x_{2} p_{2}+p_{3}
\end{gathered}
$$

IV. $\left(U_{1} U_{2}\right)=0,\left(U_{1} U_{3}\right)=0,\left(U_{2} U_{3}\right)=U_{1}$.

$$
\begin{aligned}
& p_{1}, p_{2}, x_{2} p_{1} \quad p_{1}, p_{2}, x_{2} p_{1}+x_{j} p_{2}(j>2) \quad p_{1}, p_{2}, x_{2} p_{1}+p_{3} \\
& \text { V. },\left(U_{1} U_{2}\right)=0,\left(U_{1} U_{3}\right)=0,\left(U_{2} U_{3}\right)=0 \text {. } \\
& p_{1}, p_{2}, p_{3} \quad p_{1}, p_{2}, x_{3} p_{1}+F\left(x_{3}, \ldots, x_{n}\right) p_{2} \text {. } \\
& p_{1}, x_{2} p_{1}, F\left(x_{2}, \cdots, x_{n}\right) p_{1} \quad p_{1}, p_{2}, x_{j} p_{2} \quad(j>2)
\end{aligned}
$$

§3. Normal Forms of the $G_{1}^{\prime}$ 's of Space.

It is known (see Lie's "Theorie der Transformationsgruppen," Dritter Abschnitt, Kap: 28) that the $G$ 's of $n$-dimensional space satisfy one of the structures $I, \ldots$, XII below. In each case, the $G_{4}$ contains a sub- $G_{3}$ for which we can assume one of the normal forms established in the preceding section. In the following the fourth transformation of the $G_{4}$ is determined from the fact that it must either be connected linearly with the transformations of the sub- $G_{3}$, or not so connected.

1. Suppose the transformations of the $G_{1}$ satisfy the structure

$$
\begin{aligned}
& \left(U_{1} U_{2}\right)=U_{1}, \quad\left(U_{1} U_{3}\right)=2 U_{2}, \quad\left(U_{2} U_{3}\right)=U_{3}, \quad\left(U_{i} U_{4}\right)=0 \\
& (i=1,2,3) \text {. }
\end{aligned}
$$

For the sub- $G_{3} U_{1}, U_{2}, U_{3}$ of this structure, we have found in the preceding section the following cases :

$$
p_{1}, x_{1} p_{1}, x_{1}^{2} p_{1}
$$


THE $G_{r}(r<5)$ OF $n$-DIMENGIONAL SPACE.

(ii)

$$
p_{1}, x_{1} p_{1}+x_{2} p_{2}, x_{1}^{2} p_{1}+2 x_{1} x_{2} p_{2}
$$

(iii)

$$
p_{1}, x_{1} p_{1}+x_{2} p_{2}, x_{1}^{2} p_{1}+\left(2 x_{1} x_{2}+x_{2}^{2}\right) p_{2}
$$

(iv)

$$
p_{1}, x_{1} p_{1}+x_{2} p_{2}, x_{1}^{2} p_{1}+2 x_{1} x_{2} p_{2}+x_{2} p_{3} \text {. }
$$

Further, we have for each of these cases the subdivisions

$$
U_{4} f \equiv \sum_{i=1}^{3} \rho_{i} U_{i} f ; \quad \text { or } \quad(2) U_{4} f \neq \sum_{i=1}^{3} \rho_{i} U_{i} f,
$$

the $p_{i}$ being functions of $x_{1}, \ldots, x_{n}$. We shall proceed to consider the cases and sub-cases for this structure in order.

$$
U_{1} f=p_{1}, \quad U_{2} f=x_{1} p_{1}, \quad U_{3} f=x_{1}^{2} p_{1} .
$$

(1) For this case

$$
\overleftarrow{U}_{4} f \equiv \sum_{i=1}^{3} \rho_{i} U_{i} f \equiv \xi\left(x_{1}, \cdots, x_{n}\right) p_{1}
$$

The structure shows that $\xi=0$, hence there is no $G_{4}$.

$$
U_{i} f \neq \sum_{i=1}^{3} \rho_{i} U_{i} f \equiv \sum_{i=1}^{n} \xi_{i}\left(x_{1}, \ldots, x_{n}\right) p_{i} \text {. }
$$

$U_{k} f=0(k=1, \ldots, 4)$ form a complete system of two members; hence there will be $n-2$ solutions independent of each other and of $x_{1}$. Since $U_{4} f$ is not connected linearly with $U_{1} f, \ldots, U_{3} f$, at least one other variable, $x_{2}$ (say), is not a solution. Suppose the common solutions are $\psi_{3}\left(x_{1}, \ldots, x_{n}\right), \ldots, \psi_{n}\left(x_{1}, \ldots, x_{n}\right)$. We introduce as new variables and obtain.

$$
\bar{x}_{1}=x_{1}, \quad \bar{x}_{2}=x_{2}, \quad \bar{x}_{j}=\psi_{j}\left(x_{1}, \cdots, x_{n}\right) \quad(j=3, \cdots, n),
$$

$$
\bar{U}_{1} f=\bar{p}_{1}, \quad \bar{U}_{2} f=\bar{x}_{1} \bar{p}_{1}, \quad \bar{U}_{3} f=\bar{x}_{1}^{2} \bar{p}_{1}, \quad \bar{U}_{4} f=\bar{\xi}_{1} \bar{p}_{1}+\bar{\xi}_{2} \bar{p}_{2},
$$

$\xi_{1}$ and $\xi_{2}$ being functions of $\bar{x}_{1}, \ldots, \bar{x}_{n}$. Leaving off the bars and applying the structure we find

Putting

$$
\xi_{1} \equiv 0, \quad \xi_{2} \equiv \beta\left(x_{2}, \cdots, x_{n}\right) \neq 0 .
$$

$$
\bar{x}_{2}=\int \frac{d x_{2}}{\beta}
$$


we have $\bar{U}_{1} f \equiv \bar{p}_{2}$, and thus the $G_{4}$

$$
p_{1}, x_{1} p_{1}, x_{1}^{2} p_{1}, p_{2} \text {. }
$$

(ii) $U_{1} f=p_{1}, \quad U_{2} f=x_{1} p_{1}+x_{2} p_{2}, \quad U_{3} f=x_{1}^{2} p_{1}+2 x_{1} x_{2} p_{2}$.

(1) $U_{4} f \equiv \sum_{i=1}^{3} \rho_{i} U_{i} f \equiv \xi_{1}\left(x_{1}, \ldots, x_{n}\right) p_{1}+\xi_{2}\left(x_{1}, \ldots, x_{n}\right) p_{2}$.

$\mathrm{By}$ the structure

$$
\begin{aligned}
& \left(U_{1} U_{4}\right) \equiv \xi_{1_{1}} p_{1}+\xi_{2 \bar{x}_{1}} p_{2}=0, \\
& \left(U_{2} U_{1}\right) \equiv x_{2}\left(\xi_{x_{2}} p_{1}+\xi_{2_{2}} p_{2}\right)-\xi_{1} p_{1}-\xi_{2} p_{2}=0, \\
& \left(U_{3} U_{1}\right) \equiv 2 x_{1}\left(\xi_{1} p_{1}+\xi_{2} p_{2}\right)-2 \xi_{1}\left(x_{1} p_{1}+x_{2} p_{2}\right)-2 x_{1} \xi_{2} p_{2}=0 .
\end{aligned}
$$

Whence

$$
\xi_{1}=0, \quad \xi_{2}=x_{2} \beta\left(x_{3}, \cdots, x_{n}\right) \neq 0 .
$$

Putting $x_{3}=\beta\left(x_{3}, \ldots, x_{n}\right) \neq$ const., we have the $G_{4}$

$$
p_{1}, x_{1} p_{1}+x_{2} p_{2}, x_{1}^{2} p_{1}+2 x_{1} x_{2} p_{2}, x_{2} x_{3} p_{2} \text {. }
$$

If $\beta=$ const., the $G_{4}$ has the form

$$
\frac{p_{1}, x_{1} p_{1}+x_{2} p_{2}, x_{1}^{2} p_{1}+2 x_{1} x_{2} p_{2}, x_{2} p_{2}}{U_{i} f \neq \sum_{i=1}^{3} U_{i} f .}
$$

As under (i), we can introduce new variables so that

$$
U_{4} f \equiv \xi_{1}\left(x_{1}, \cdots, x_{n}\right) p_{1}+\xi_{2}\left(x_{1}, \cdots, x_{n}\right) p_{2}+\xi_{3}\left(x_{1}, \ldots, x_{n}\right) p_{3} \text {. }
$$

By the structure

$$
\xi_{1} \equiv 0, \quad \xi_{2} \equiv x_{2} \beta\left(x_{3}, \cdots, x_{n}\right), \quad \xi_{3} \equiv \gamma\left(x_{3}, \cdots, x_{n}\right) \neq 0 .
$$

Introducing

$$
\bar{x}_{2}=x_{2} \phi\left(x_{3}, \cdots, x_{n}\right), \quad \bar{x}_{3}=\int \frac{d x_{3}}{\gamma},
$$

and choosing $\phi$ so that $\beta \phi+\gamma \phi_{x_{3}}=0$, we have

$$
\bar{U}_{4} f=\bar{p}_{3} \text {. }
$$

Thus for this case we get the group

$$
p_{1}, x_{1} p_{1}+x_{2} p_{2}, x_{1}^{2} p_{1}+2 x_{1} x_{2} p_{2}, p_{3} \text {. }
$$


(iii) $U_{1} f=p_{1}, U_{2} f=x_{1} p_{1}+x_{2} p_{2}, U_{3} f=x_{1}^{2} p_{1}+\left(2 x_{1} x_{2}+x_{2}^{2}\right) p_{2}$.

(1) $U_{4} f \equiv \sum_{i=1}^{3} \rho_{i} U_{i} f \equiv \xi_{1}\left(x_{1}, \cdots, x_{n}\right) p_{1}+\xi_{2}\left(x_{1}, \ldots, x_{n}\right) p_{2}$.

The structure gives the conditions

$$
\begin{aligned}
& \left(U_{1} U_{1}\right) \equiv \xi_{1_{1}} p_{1}+\xi_{2 x_{1}} p_{2}=0, \\
& \left(U_{2} U_{4}\right) \equiv x_{2}\left(\xi_{x_{3}} p_{1}+\xi_{2 x_{3}} p_{2}\right)-\xi_{1} p_{1}-\xi_{2} p_{2}=0, \\
& \left(U_{3} U_{4}\right) \equiv\left(2 x_{1}+x_{2}\right)\left(\xi_{1} p_{1}+\xi_{2} p_{2}\right)-2 \xi_{1}\left(x_{1} p_{1}+x_{2} p_{2}\right) \\
& \quad-2 x_{1} \xi_{2} p_{2}-2 x_{2} \xi_{2} p_{2}=0 .
\end{aligned}
$$

From the third relation $\xi_{1}=\xi_{2}=0$; hence there is no $G_{1}$ for this case.

$$
U_{4} f \neq \sum_{i=1}^{3} \rho_{i} U_{i} f
$$

As before, we can introduce new variables so that

$$
U_{1} f \equiv \xi_{1}\left(x_{1}, x_{n}\right) p_{1}+\xi_{2}\left(x_{1}, \cdots, x_{n}\right) p_{2}+\xi_{3}\left(x_{1}, \ldots, x_{n}\right) p_{3} \text {. }
$$

Hence by the structure,

$$
\begin{aligned}
& \left(U_{1} U_{4}\right) \equiv \xi_{x_{1}} p_{1}+\xi_{2 x_{1}} p_{2}+\xi_{x_{1}} p_{3}=0, \\
& \left(U_{2} U_{1}\right) \equiv x_{2}\left(\xi_{1_{1}} p_{1}+\xi_{x_{2}} p_{2}+\xi_{x_{3}} p_{3}\right)-\xi_{1} p_{1}-\xi_{2} p_{2}=0, \\
& \left(U_{3} U_{4}\right) \equiv\left(2 x_{1}+x_{2}\right)\left(\xi_{1} p_{1}+\xi_{2} p_{2}\right)-2 \xi_{1}\left(x_{1} p_{1}+x_{2} p_{2}\right)-2\left(x_{1}+x_{2}\right) \xi_{2} p_{2}=0 .
\end{aligned}
$$

From these relations

Introducing

$$
\xi_{1}=\xi_{2}=0, \quad \xi_{3} \equiv \gamma\left(x_{3}, \cdots, x_{n}\right) \neq 0 .
$$

we have $\bar{U}_{1} f \equiv \bar{p}_{3}$.

$$
\bar{x}_{9}=\int \frac{d x_{3}}{\gamma}
$$

Hence we find the $G_{4}$ :

(iv) $U_{1} f=p_{1}, U_{2} f=x_{1} p_{1}+x_{2} p_{2}, U_{3} f=x_{1}^{2} p_{1}+2 x_{1} x_{2} p_{2}+x_{2} p_{3}$.

$$
U_{1} f \equiv \sum_{i=1}^{3} p_{i} U_{i} f \equiv \sum_{i=1}^{3} \xi_{i}\left(x_{1}, \cdots, x_{n}\right) p_{i}
$$


WILLIAM B. STONE.

The structure gives the conditions

$\left(U_{1} U_{4}\right) \equiv \xi_{1_{x_{1}}} p_{1}+\xi_{2_{x_{1}}} p_{2}+\xi_{3_{x_{1}}} p_{3}=0$,

$\left(D_{2} U_{4}\right) \equiv x_{2}\left(\xi_{1_{2}} p_{1}+\xi_{x_{2}} \cdot p_{2}+\xi_{x_{2}} \cdot p_{3}\right)-\xi_{1} p_{1}-\xi_{2} p_{2}=0$ ，

$\left(U_{3} U_{1}\right) \equiv 2 x_{1}\left(\xi_{1} p_{1}+\xi_{2} p_{2}\right)+x_{2}\left(\xi_{1_{3}} p_{1}+\xi_{2 x_{3}} p_{2}+\xi_{3 x_{3}} p_{3}\right)$

Whence

$$
-2 \xi_{1}\left(x_{1} p_{1}+x_{2} p_{2}\right)-\xi_{2}\left(2 x_{1} p_{2}+p_{3}\right)=0 \text {. }
$$

$\xi_{1} \equiv x_{2} \alpha\left(x_{1}, \cdots, x_{n}\right), \xi_{2}=2 x_{2} x_{3} \alpha\left(x_{1}, \cdots, x_{n}\right)+x_{2} \beta\left(x_{1}, \cdots, x_{n}\right)$,

$\xi_{3} \equiv x_{3}^{2} \alpha\left(x_{4}, \cdots, x_{n}\right)+x_{3} \beta\left(x_{4}, \cdots, x_{n}\right)+\gamma\left(x_{4}, \ldots, x_{n}\right)$,

so that

(a)

$$
U_{4} f \equiv x_{2} \alpha p_{1}+\left(2 x_{2} x_{3} \alpha+\beta\right) p_{2}+\left(x_{3}^{2} \alpha+x_{3} \beta+\gamma\right) p_{3} .
$$

Introduce

$$
\alpha\left(x_{1}, \cdots, x_{n}\right) \neq 0 \text {. }
$$

$$
\bar{x}_{2}=x_{2} \alpha\left(x_{1}, \cdots, x_{n}\right), \quad \bar{x}_{3}=x_{3} \alpha\left(x_{1}, \cdots, x_{n}\right) .
$$

Then (omiting the bars)

Introduce again

$$
U_{4} f=x_{2} p_{1}+2 x_{2}\left(x_{3}+\frac{\beta}{2}\right) p_{2}+\left(x_{3}^{2}+x_{3} \beta+\alpha \gamma\right) p_{3} .
$$

Hence

$$
\bar{x}_{3}=x_{3}+\frac{\beta}{2}, \quad \bar{x}_{4}=\alpha \cdot \gamma-\frac{\beta^{2}}{4} \neq \text { const. }
$$

$p_{1}, x_{1} p_{1}+x_{2} p_{2}, x_{1}^{2} p_{1}+2 x_{1} x_{2} p_{2}+x_{2} p_{3}, x_{2} p_{1}+2 x_{2} x_{3} p_{2}+\left(x_{3}^{2}+x_{4}\right) p_{3}$.

If $\alpha \gamma-\beta^{2} / 4=$ const., we have

$p_{1}, x_{1} p_{1}+x_{2} p_{2}, x_{1}^{2} p_{1}+2 x_{1} x_{2} p_{2}+x_{2} p_{3}, x_{2} p_{1}+2 x_{2} x_{3} p_{2}+x_{3}^{2} p_{3}$.

(b)

$$
\alpha\left(x_{1}, \cdots, x_{n}\right)=0, \beta\left(x_{1}, \cdots, x_{n}\right) \neq 0 .
$$

Introduce

Then

$$
\bar{x}_{2}=x_{2} \beta, \quad \bar{x}_{3}=x_{3} \beta+\gamma, \quad \bar{x}_{4}=\beta \neq \text { const. }
$$

and we get the $G_{\text {, }}$

$$
\bar{U}_{4} f \equiv \bar{x}_{2} \bar{x}_{4} \bar{p}_{2}+\bar{x}_{3} \bar{x}_{4} \bar{p}_{3} \text {, }
$$

$$
p_{1}, x_{1} p_{1}+x_{2} p_{2}, x_{1}^{2} p_{1}+2 x_{1} x_{2} p_{2}+x_{2} p_{3}, x_{1}\left(x_{2} p_{2}+x_{3} p_{3}\right) \text {. }
$$


THE $G_{r}(r<5)$ OF $n$-DIMENSIONAL SPACE.

If $\beta=$ const. we have

$$
p_{1}, x_{1} p_{1}+x_{2} p_{2}, x_{1}^{2} p_{1}+2 x_{1} x_{2} p_{2}+x_{2} p_{3}, x_{2} p_{2}+x_{3} p_{3} \text {. }
$$

(c) If $\beta$ is also zero, $\gamma \neq 0$. Then introduce $\overline{x_{2}}=x_{2} / \gamma, \bar{x}_{3}=x_{3} / \gamma$, and we have the $G_{1}$

$$
\frac{p_{1}, x_{1} p_{1}+x_{2} p_{2}, x_{1}^{2} p_{1}+2 x_{1} x_{2} p_{2}+x_{2} p_{3}, p_{3}}{U_{4} f \neq \sum_{i=1}^{3} \rho_{i} U_{i} f .}
$$

As before, we can introduce new variables so that

$$
U_{1} f \equiv \sum_{i=1}^{4} \xi_{i}\left(x_{1}, \cdots, x_{n}\right) p_{i}
$$

By the structure we get, as in the preceding case,

$$
U_{4} f \equiv x_{2} \alpha p_{1}+2 x_{2}\left(x_{3} \alpha+\frac{\beta}{2}\right) p_{2}+\left(x_{3}^{2} \alpha+x_{3} \beta+\gamma\right) p_{3}+\delta p_{4},
$$

$\alpha, \beta, \gamma, \delta$ being functions of $x_{1}, \cdots, x_{n}$.

$\delta$ cannot be zero, so that we can assume that the coefficient of $p_{t}$ has been made unity by the substitution

Then

$$
\bar{x}_{1}=\int \frac{d x_{4}}{\delta}
$$

$$
U_{1} f \equiv x_{2} \alpha p_{1}+2 x_{2}\left(x_{3} \alpha+\frac{\beta}{2}\right) p_{2}+\left(x_{3}^{2} \alpha+x_{j} \beta+\gamma\right) p_{3}+p_{1}
$$

Now by assuming $\alpha=0$, which is certainly one value the arbitrary function $\alpha$ may have, and by introducing as new variables

we find

$$
\bar{x}_{2}=x_{2} \epsilon^{-\int \beta d x_{4}}, \vec{x}_{3}=x_{3} \epsilon^{-\int \beta d x_{4}}-\int \gamma \epsilon^{-\int \beta d x_{4}} \cdot d x_{4},
$$

Thus we get the group

$$
\bar{U}_{1} f=p_{\imath} \cdot
$$

$$
p_{1}, x_{1} p_{1}+x_{2} p_{2}, x_{1}^{2} p_{1}+2 x_{1} x_{2} p_{2}+x_{2} p_{3}, p_{1} \text {. }
$$

This group is simply transitive in the four variables $x_{1}, \ldots, x_{1}$. Hence by Lie's "Theorie der Transformationsgruppen" (Vol. I, 
WILLTAMI B. STONE.

page 340 ), all types under this structure and subdivision that arise when $\alpha \neq 0$ and $n=4$ can be transformed into the above given form. Moreover when $n>4$, the variables $x_{5}, \ldots, x_{n}$ play the rôle of constants. Hence all $G_{1}$ 's belonging to this type $-i$. e., which have this structure and are simply transitive with regard to $x_{1}, \ldots, x_{4}-$ can be transformed into the above $G_{1}$ by a proper choice of variables.

II. Let us consider next the groups satisfying the structure

$$
\begin{gathered}
\left(U_{1} U_{2}\right)=\left(U_{1} U_{3}\right)=\left(U_{2} U_{1}\right)=\left(U_{3} U_{4}\right)=0 \\
\left(U_{2} U_{3}\right)=U_{2}, \quad\left(U_{1} U_{1}\right)=U_{1} .
\end{gathered}
$$

For the sub- $G_{3}, U_{1}, U_{2}, U_{3}$, we having the following cases:

$$
\begin{gathered}
p_{1}, p_{2}, x_{2} p_{2} \\
\frac{p_{1}, x_{2} p_{1},-x_{2} p_{2}}{p_{1}, p_{2}, x_{3} p_{1}+x_{2} p_{2}} \\
p_{1}, p_{2}, x_{2} p_{2}+p_{3}
\end{gathered}
$$

(1) We will consider first all the cases for which

$$
U_{4} f \equiv \sum_{i=1}^{3} \rho_{i}\left(x_{1}, \cdots, x_{n}\right) U_{i} f
$$

(i) For the case (i) above,

$$
U_{1} f \equiv \xi_{1}\left(x_{1}, \cdots, x_{n}\right) p_{1}+\xi_{2}\left(x_{1}, \ldots, x_{n}\right) p_{2} .
$$

By the structure, we find immediately

$$
\xi_{1} \equiv x_{1}+\alpha\left(x_{3}, \cdots, x_{n}\right), \quad \xi_{3} \equiv 0 .
$$

Putting $\bar{x}_{1}=x_{1}+\alpha$, we obtain the $G_{1}$,

(ii) Likewise here

$$
p_{1}, p_{2}, x_{2} p_{2}, x_{1} p_{1} \text {. }
$$

$$
U_{4} f \equiv \xi_{1}\left(x_{1}, \cdots, x_{n}\right) p_{1}+\xi_{2}\left(x_{1}, \cdots, x_{n}\right) p_{2} .
$$

The structure gives $\xi_{1} \equiv x_{1}+\alpha\left(x_{3}, \cdots, x_{n}\right), \xi_{2} \equiv x_{2}$. Hence putting $\bar{x}_{1}=x_{1}+\alpha$, we get the $G_{4}$, 


$$
p_{1}, x_{2} p_{1},-x_{2} p_{2}, x_{1} p_{1}+x_{2} p_{2} \text {. }
$$

(iii) The structure gives no new $G_{4}$ for this case.

(iv) For this case

By the structure we find

$$
U_{4} f \equiv \sum_{i=1}^{3} \xi_{i}\left(x_{1}, \cdots, x_{n}\right) p_{i} .
$$

$\xi_{1} \equiv x_{1}+\alpha\left(x_{4}, \cdots, x_{n}\right), \quad \xi_{2} \equiv \epsilon^{x_{3}+\beta\left(x_{1}, \ldots, x_{n}\right)}, \quad \xi_{3} \equiv \gamma\left(x_{4}, \cdots, x_{n}\right)$. If $\alpha$ and $\beta$ are not zero, they can be made to disappear by the new variables

$$
\bar{x}_{1}=x_{1}+\alpha, \quad \bar{x}_{3}=x_{3}+\beta, \quad \bar{x}_{4}=\gamma\left(x_{4}, \cdots, x_{n}\right) \neq \text { const. }
$$

Thus we obtain the $G_{4}$

$$
p_{1}, p_{2}, x_{2} p_{2}+p_{3}, x_{1} p_{1}+\epsilon^{x_{8}} p_{2}+x_{4} p_{3} \text {. }
$$

If $\gamma=$ const., we have

$$
p_{1}, p_{2}, x_{2} p_{2}+p_{3}, x_{1} p_{1}+\epsilon^{x_{3}} p_{2}+c p_{3} \text {. }
$$

(2) We will consider next the cases for which

$$
U_{4} f \neq \sum_{i=1}^{3} \rho_{i} U_{i} f \equiv \sum_{i=1}^{n} \xi_{i}\left(x_{1}, \cdots, x_{n}\right) p_{i} .
$$

(i) In this case $U_{k} f=0(k=1, \ldots, 4)$ form a complete system of 3 independent members; hence there will be $n-3$ common solutions, $\psi_{1}\left(x_{1}, \cdots, x_{n}\right), \ldots, \psi_{n}\left(x_{1}, \cdots, x_{n}\right)$, independent of each other and of $x_{1}, x_{2}$, and at least one of the remaining variables, $x_{3}$ (say). Then we can introduce as a new system of variables

$$
\bar{x}_{1}=x_{1}, \quad \bar{x}_{2}=x_{2}, \quad \bar{x}_{3}=x_{3}, \quad \bar{x}_{j}=\psi_{j}\left(x_{1}, \cdots, x_{n}\right), \quad(j=4, \cdots, n) \text {. }
$$

By these $U_{1}, U_{2}, U_{3}$ remain unchanged, and

$$
\bar{U}_{1} f \equiv \bar{\xi}_{1} \bar{p}_{1}+\bar{\xi}_{2} \bar{p}_{2}+\bar{\xi}_{3} \bar{p}_{3} \text {, }
$$

$\xi_{i}$ being functions of the new variables $\vec{x}_{1}, \ldots, \bar{x}_{n^{*}}$ From the structure we find immediately

$$
U_{1} f \equiv x_{1} p_{1}+p_{3}
$$


and thus we have the $G_{4}$

$$
p_{1}, p_{2}, x_{2} p_{2}, x_{1} p_{1}+p_{3} \text {. }
$$

(ii) As in the preceding case we have

$$
U_{\downarrow} f=\sum_{i=1}^{3} \xi_{i}\left(x_{1}, \cdots, x_{n}\right) p_{i}
$$

and by the structure we find

Hence the $G_{4} \begin{gathered}U_{1} f \equiv x_{1} p_{1}+x_{2} p_{2}+p_{3} . \\ p_{1}, x_{2} p_{1}, x_{2} p_{2}, x_{1} p_{1}+x_{2} p_{2}+p_{3}\end{gathered}$.

(iii) For this case we have to make two subdivisions, according as $\xi_{3}=0$ or $\xi_{3} \neq 0$.

(a) $\xi_{3} \neq 0$. Then $x_{3}$ is not a common solution of the complete system, and we can introduce, as above,

Then

$$
\bar{x}_{1}=x_{1}, \quad \bar{x}_{2}=x_{2}, \quad \bar{x}_{3}=x_{3}, \quad \bar{x}_{j}=\psi_{j} \quad(j=4, \cdots, n) .
$$

and we obtain the $G_{4}$

$$
\bar{U}_{4} f \equiv \sum_{i=1}^{3} \xi_{i}\left(x_{1}, \cdots, x_{n}\right) p_{i}
$$

$$
p_{1}, p_{2}, x_{3} p_{1}+x_{2} p_{2}, x_{1} p_{1}+x_{3} p_{3} \text {. }
$$

(b) $\xi_{3}=0$. For this case $x_{3}$ is a common solution of the complete system, and at least one of the other variables $x_{1}, \ldots, x_{n}$ is not a.solution (say, $x_{4}$ ). Then we can introduce as a new system of variables

$$
\bar{x}_{1}=x_{1}, \quad \bar{x}_{2}=x_{2}, \quad \bar{x}_{4}=x_{4}, \quad \bar{x}_{3}=x_{3}, \quad \bar{x}_{j}=\psi_{j} \quad(j=5, \cdots, n),
$$

the first three because they are not solutions, and the last $n-3$ because they are independent common solutions. Thus we get

$$
\bar{U}_{4} f \equiv \bar{\xi}_{1}\left(\bar{x}_{1}, \cdots, \bar{x}_{n}\right) \bar{p}_{1}+\bar{\xi}_{2}\left(\bar{x}_{1}, \ldots, \bar{x}_{n}\right) \bar{p}_{2}+\bar{\xi}_{4}\left(\bar{x}_{1}, \ldots, x\right) \bar{p}_{4} .
$$

Applying the structure, we find

$$
\begin{aligned}
& \left(U_{1} U_{4}\right) \equiv \xi_{1_{1}} p_{1}+\xi_{2 x_{1}} p_{2}+\xi_{1_{x_{1}}} p_{4}=p_{1} \\
& \left(U_{2} U_{4}\right) \equiv \xi_{1_{3}} p_{1}+\xi_{2 x_{2}} p_{2}+\xi_{x_{2}} p_{4}=0 \\
& \left(U_{3} U_{4}\right) \equiv x_{3} p_{1}-\xi_{2} p_{2}=0
\end{aligned}
$$


THE $G_{r}(r<5)$ OF $n$-DIMENSIONAL SPACE.

Hence this case holds only when $x_{3}=0$, which is impossible.

(iv) For this case

$$
U_{4} f \equiv \sum_{i=1}^{4} \xi_{i}\left(x_{1}, \cdots, x_{n}\right) y_{i} .
$$

The structure gives the conditions

Whence

$$
\begin{aligned}
\left(U_{1} U_{4}\right) & \equiv \sum_{i=1}^{4} \xi_{i_{1}} p_{i}=p_{1}, \\
\left(U_{2} U_{1}\right) & \equiv \sum_{i=1}^{4} \xi_{i_{3}} p_{i}=0 \\
\left(U_{3} U_{4}\right) & \equiv \sum_{i=1}^{4} \xi_{i x_{3}} p_{i}-\xi_{2} \cdot p_{2}=0 .
\end{aligned}
$$

$$
\begin{array}{ll}
\xi_{1} \equiv x_{1}+\alpha\left(x_{1}, \cdots, x_{n}\right), & \xi_{2} \equiv \beta\left(x_{4}, \cdots, x_{n}\right) \epsilon^{x_{3}}, \\
\xi_{3} \equiv \gamma\left(x_{1}, \cdots, x_{n}\right), & \xi_{4} \equiv \delta\left(x_{4}, \cdots, x_{n}\right) .
\end{array}
$$

$\xi_{3}$ can be made zero and $\xi_{4}$ can be made unity by the introduction of new variables. Algo if we assume $\beta \equiv 1$, we get a $G_{1}$, to which, being simply transitive in four variables, all groups under this case will be similar, as on page 22. Hence we obtain the $G_{\text {, }}$

$$
p_{1}, p_{2}, x_{2} p_{2}+p_{3}, x_{1} p_{1}+\epsilon^{x_{3}} p_{2}+p_{4} \text {. }
$$

III. Suppose the four transformations satisfy the structure

$$
\begin{aligned}
& \left(U_{1} U_{2}\right)=0, \quad\left(U_{1} U_{3}\right)=0, \quad\left(U_{2} U_{3}\right)=U_{1}, \\
& \left(U_{1} U_{1}\right)=c U_{1},\left(U_{2} U_{1}\right)=U_{2},\left(U_{3} U_{4}\right)=(c-1) U_{3}, \quad(c \neq 1) .
\end{aligned}
$$

For the sub- $G_{3}, U_{1}, U_{2}, U_{3}$, we found in $\$ 2$ these cases :

(i)

$$
p_{1}, p_{2}, x_{2} p_{1}
$$

(ii)

$$
p_{1}, p_{2}, x_{2} p_{1}+x_{3} p_{2}
$$

(iii)

$$
p_{1}, p_{2}, x_{2} p_{1}+p_{3} \text {. }
$$

To these will be added

$$
p_{1}, x_{2} p_{1},-p_{2} \text {, }
$$

which as a $G_{3}$ is the same as (i), but these two give rise to different $G_{1}^{\prime}$ 's. 
(1) As before we will consider first all the cases for which

(i) For this case

$$
U_{4} f \equiv \sum_{i=1}^{3} \rho_{i} U_{i} f
$$

$$
U_{1} f \equiv \xi_{1}\left(x_{1}, \ldots, x_{n}\right) p_{1}+\xi_{2}\left(x_{1}, \ldots, x_{n}\right) p_{2} .
$$

From the structure we find immediately

$$
\xi_{1} \equiv c x_{1}+\alpha\left(x_{3}, \cdots, x_{n}\right), \quad \xi_{2} \equiv x_{2} .
$$

(a) If $c \neq 0$, put $\bar{x}_{1}=c x_{1}+\alpha$, and we have the group

$$
p_{1}, p_{2}, x_{2} p_{1}, c x_{1} p_{1}+x_{2} p_{2} \text {. }
$$

(b) If $c=0$, put $\bar{x}_{3}=\alpha$, and we have

(ii) Here also

$$
p_{1}, p_{2}, x_{2} p_{1}, x_{3} p_{1}+x_{2} p_{2} \text {. }
$$

$$
U_{1} f \equiv \xi_{1}\left(x_{1}, \ldots, x_{n}\right) p_{1}+\xi_{2}\left(x_{1}, \ldots, x_{n}\right) p_{2} .
$$

We-find-from the structure that $c=2$ for this case to hold. Then follows that

$$
\xi_{1} \equiv 2 x_{1}+\alpha\left(x_{3}, \cdots, x_{n}\right), \quad \xi_{2} \equiv x_{2} .
$$

Putting $\bar{x}_{1}=x_{1}+\alpha / 2$, we obtain the group

$$
p_{1}, p_{2}, x_{2} p_{1}+x_{3} p_{2}, 2 x_{1} p_{1}+x_{2} p_{2} \text {. }
$$

(iii) For this case

$$
U_{4} f \equiv \xi_{1}\left(x_{1}, \cdots, x_{n}\right) p_{1}+\xi_{2}\left(x_{1}, \cdots, x_{n}\right) p_{2}+\xi_{3}\left(x_{1}, \ldots, x_{n}\right) p_{3} .
$$

Substituting in the structure, we find

$$
\xi_{1} \equiv c x_{1}+\alpha\left(x_{3}, \cdots, x_{n}\right), \quad \xi_{3} \equiv(c-1) x_{3}+\gamma\left(x_{4}, \cdots, x_{n}\right),
$$

Whence

$$
\xi_{2} \equiv x_{2}+\alpha_{x_{3}} \equiv x_{2}+\beta\left(x_{1}, \cdots, x_{n}\right) \text {. }
$$

$$
\alpha \equiv x_{3} \beta+\lambda\left(x_{1}, \cdots, x_{n}\right) \text {. }
$$

$\lambda$ and $\gamma$ can be made to disappear in the usual way.

Then introduce as new variables

$$
\bar{x}_{1}=x_{1}+\beta\left(x_{4}, \cdots, x_{n}\right) \cdot x_{3}, \quad \bar{x}_{2}=x_{2}+\beta\left(x_{4}, \cdots, x_{n}\right) .
$$


THE $G_{r}(r<5)$ OF $n$-DIMENSIONAL SPACE.

Thus we obtain the group

$$
p_{1}, p_{2}, x_{2} p_{1}+p_{3}, c x_{1} p_{1}+x_{2} p_{2}+(c-1) x_{3} p_{3} \text {. }
$$

(iv) For this case

$$
U_{1} f \equiv \xi_{1}\left(x_{1}, \cdots, x_{n}\right) p_{1}+\xi_{2}\left(x_{1}, \cdots, x_{n}\right) p_{2} .
$$

By the structure we find immediately

$$
\xi_{1} \equiv c x_{1}+\alpha\left(x_{3}, \cdots, x_{n}\right), \quad \xi_{2} \equiv(c-1) x_{2} .
$$

(a) $c \neq 0$. Introduce $c \bar{x}_{1}=c x_{1}+\alpha\left(x_{3}, \cdots, x_{n}\right)$. Then we have

$$
p_{1}, x_{2} p_{1},-p_{2}, c x_{1} p_{1}+(c-1) x_{2} p_{2} \text {. }
$$

(b) $c=0$. Put $\bar{x}_{3}=\alpha\left(x_{3}, \cdots, x_{n}\right) \neq$ const. Thus

$$
p_{1}, x_{2} p_{1},-p_{2}, x_{3} p_{1}-x_{2} p_{2} \text {. }
$$

(c) $c=0, \alpha=$ const. . Then we have

$$
p_{1}, x_{2} p_{1}, p_{2}, p_{1}-x_{2} p_{2} \text {. }
$$

(2) We will next consider all the cases for which

$$
U_{\downarrow} f \neq \sum_{i=1}^{3} \rho_{i} U_{i} f \equiv \sum_{i=1}^{n} \xi_{i}\left(x_{1}, \cdots, x_{n}\right) p_{i}
$$

(i) For this case we can introduce new variables so that

$$
U_{4} f \equiv \sum_{i=1}^{3} \xi_{i}\left(x_{1}, \cdots, x_{n}\right) p_{i}
$$

Applying the structure and reducing in the usual way, we obtain the group

$$
p_{1}, p_{2}, x_{2} p_{1}, c x_{1} p_{1}+x_{2} p_{2}+p_{3} \text {. }
$$

(ii) Here we have to make two cases according as

$$
\xi_{3}=0 \text { or } \xi_{3} \neq 0 \text {. }
$$

(a) $\xi_{3} \neq 0$. Then since there are three independent members of the complete system, obtained by writing $U_{i} f=0$, there will be $n-3$ common solutions, independent of $x_{1}, x_{2}, x_{3}$, and of each other. Suppose these solutions are $\psi_{4}\left(x_{1}, \cdots, x_{n}\right), \cdots, \psi_{n}\left(x_{1}, \cdots, x_{n}\right)$. 
Then we can introduce as new variables

$$
\bar{x}_{1}=x_{1}, \quad \bar{x}_{2}=x_{2}, \quad \bar{x}_{3}=x_{3}, \quad \bar{x}_{j}=\psi_{j}\left(x_{1}, \cdots, x_{n}\right) \quad(j=4, \cdots, n) \text {, }
$$
and thus obtain

$$
\bar{U}_{4} f \equiv \xi_{1}\left(\bar{x}_{1}, \ldots, \bar{x}_{n}\right) \bar{p}_{1}+\xi_{2}\left(\bar{x}_{1}, \ldots, \bar{x}_{n}\right) \bar{p}_{2}+\xi_{3}\left(\bar{x}_{1}, \ldots, \bar{x}_{n}\right) \bar{p}_{3} .
$$

Applying the structure we find

$$
U_{4} f \equiv c x_{1} p_{1}+x_{2} p_{2}+(2-c) x_{3} p_{3}
$$

and thus we have the $G_{4}$

$$
p_{1}, p_{2}, x_{2} p_{1}+x_{3} p_{2}, c x_{1} p_{1}+x_{2} p_{2}+(2-c) x_{3} p_{3} \text {. }
$$

(b) $\xi_{3}=0$. In this case $x_{3}$ will be a solution, and there will be only $n-4$ others, $\psi_{5}\left(x_{1}, \ldots, x_{n}\right), \ldots, \psi_{n}\left(x_{1}, \cdots, x_{n}\right)$. At least one of the remaining variables, $x_{4}$ (say), must not be a solution for this case to hold. Hence we can introduce as new variables $\bar{x}_{1}=x_{1}, \quad \bar{x}_{2}=x_{2}, \quad \bar{x}_{1}=x_{4}, \quad \bar{x}_{3}=x_{3}, \quad \bar{x}_{j}=\psi_{1}\left(x_{1}, \cdots, x_{n}\right) \quad(j=5, \cdots, n)$. Then

$$
\bar{U}_{4} f \equiv \bar{\xi}_{1}\left(\bar{x}_{1}, \cdots, \bar{x}_{n}\right) \bar{p}_{1}+\bar{\xi}_{2}\left(\bar{x}_{1}, \ldots, \bar{x}_{n}\right) \bar{p}_{2}+\xi_{4}\left(\bar{x}_{1}, \ldots, \bar{x}_{n}\right) \bar{p}_{1} .
$$

From the structure we find, leaving off the bars,

$$
\xi_{1} \equiv 2 x_{1}+\alpha\left(x_{3}, \cdots, x_{n}\right), \quad \xi_{2} \equiv x_{2}, \quad \xi_{1} \equiv \gamma\left(x_{3}, \ldots, x_{n}\right) \neq 0 .
$$

This shows $c=2$ for this case.

Now introduce

$$
\bar{x}_{1}=x_{1}+\phi\left(x_{3}, \cdots, x_{n}\right), \quad \bar{x}_{1}=\int \frac{d x_{1}}{\gamma},
$$
and choosing $\phi$ so as to make the arbitrary function in $\xi_{1}$ disappear,
we find

Thus the $G_{1}$

$$
U_{1} f \equiv 2 x_{1} p_{1}+x_{2} p_{2}+p_{1}
$$

$$
p_{1}, p_{2}, x_{2} p_{1}+x_{3} p_{2}, 2 x_{1} p_{1}+x_{2} p_{2}+p_{4} \text {. }
$$

(iii) We can introduce new variables directly so that

$$
U_{i} f \equiv \sum_{i=1}^{4} \xi_{i}\left(x_{1}, \ldots, x_{n}\right) p_{i}
$$


THE $G_{r}(r<5)$ OF $n$-DIMENGIONAL SPAOE.

From the structure, we find immediately

$$
\begin{array}{ll}
\xi_{1} \equiv c x_{1}+\alpha\left(x_{3}, \cdots, x_{n}\right), & \xi_{2} \equiv x_{2}+\beta\left(x_{1}, \cdots, x_{n}\right), \\
\xi_{3} \equiv(c-1) x_{3}+\gamma\left(x_{4}, \cdots, x_{n}\right), & \xi_{4} \equiv \delta\left(x_{1}, \cdots, x_{n}\right) \neq 0 .
\end{array}
$$

$\xi_{4}$ can be made unity by the substitution

$$
\bar{x}_{4} \equiv \int \frac{d x_{4}}{\delta}
$$

and $\gamma$ can be made to vanish in the usual way. Now if $\alpha$ and $\beta$, which are arbitrary, have the value zero, we obtain the group, which is simply transitive in four variables

$$
p_{1}, p_{2}, x_{2} p_{1}+p_{3}, c x_{1} p_{1}+x_{2} p_{2}+(c-1) x_{3} p_{3}+p_{4} \text {. }
$$

To this group, being simply transitive, every group under this case, when $n=4$, must be similar, whatever be the forms of the functions $\alpha$ and $\beta$, according to Lie's "Theorie der Transformationsgruppen," (Vol. I, page 340). The remaining variables $x_{b}, \cdots, x_{n}$ play the rôle of constants in the arbitrary functions, and do not affect the equations of transformations in four variables, that would transform any type into the one above. Hence all $G_{4}$ 's belonging to this type can be transformed into the above-given $G_{1}$.

(iv) Finally for this case, we can introduce new variables so that

$$
U_{1} f \equiv \sum_{i=1}^{3} \rho_{i} U_{i} f
$$

Applying the structure and reducing in the usual way, we obtain the group

$$
p_{1}, x_{2} p_{1}, p_{2}, c x_{1} p_{1}+(c-1) x_{2} p_{2}+p_{3} \text {. }
$$

The treatment for the remaining structures is very similar to the preceding ; we will therefore not give the calculations in full, but will in concluding this section give the results in a complete table of all $G$ 's, classified according to their structures.

I. $\left(U_{1} U_{2}\right)=U_{1}, \quad\left(U_{1} U_{3}\right)=2 U_{2}, \quad\left(U_{2} U_{3}\right)=U_{3}, \quad\left(U_{i} U_{k}\right)=0$ $(i, k,=1,2,3)$. 
30

WILLIAM B. STONE.

$$
\begin{aligned}
& p_{1}, x_{1} p_{1}, x_{1}^{2} p_{1}, p_{2} \quad p_{1}, x_{1} p_{1}+x_{2} p_{2}, x_{1}^{2} p_{1}+2 x_{1} x_{2} p_{2}, x_{2} x_{3} p_{2} \\
& p_{1}, x_{1} p_{1}+x_{2} p_{2}, x_{1}^{2} p_{1}+2 x_{1} x_{2} p_{2}, x_{2} p_{2} \\
& p_{1}, x_{1} p_{1}+x_{2} p_{2}, x_{1}^{2} p_{1}+2 x_{1} x_{2} p_{2}, p_{3} \\
& p_{1}, x_{1} p_{1}+x_{2} p_{2}, x_{1}^{2} p_{1}+\left(2 x_{1} x_{2}+x_{2}^{2}\right) p_{2}, p_{3} \\
& p_{1}, x_{1} p_{1}+x_{2} p_{2}, x_{1}^{2} p_{1}+2 x_{1} x_{2} p_{2}+x_{2} p_{3}, x_{2} p_{1}+2 x_{2} x_{3} p_{2}+\left(x_{3}^{2}+x_{4}\right) p_{3} \\
& p_{1}, x_{1} p_{1}+x_{2} p_{2}, x_{1}^{2} p_{1}+2 x_{1} x_{2} p_{2}+x_{2} p_{3}, x_{2} p_{1}+2 x_{2} x_{3} p_{2}+x_{3}^{2} p_{3} \\
& p_{1}, x_{1} p_{1}+x_{2} p_{2}, x_{1}^{2} p_{1}+2 x_{1} x_{2} p_{2}+x_{2} p_{3}, x_{4}\left(x_{2} p_{2}+x_{3} p_{3}\right) \\
& p_{1}, x_{1} p_{1}+x_{2} p_{2}, x_{1}^{2} p_{1}+2 x_{1} x_{2} p_{2}+x_{2} p_{3}, x_{2} p_{2}+x_{3} p_{3} \\
& p_{1}, x_{1} p_{1}+x_{2} p_{2}, x_{1}^{2} p_{1}+2 x_{1} x_{2} p_{2}+x_{2} p_{3}, p_{3} \\
& p_{1}, x_{1} p_{1}+x_{2} p_{2}, x_{1}^{2} p_{1}+2 x_{1} x_{2} p_{2}+x_{2} p_{3}, p_{1} \\
& \text { II. }\left(U_{1} U_{2}\right)=\left(U_{1} U_{3}\right)=\left(U_{2} U_{4}\right)=\left(U_{3} U_{4}\right)=0,\left(U_{2} U_{3}\right)=U_{2} \text {, } \\
& \left(U_{1} U_{4}\right)=U_{1} \\
& p_{1}, p_{2}, x_{2} p_{2}, x_{1} p_{1} \quad p_{1}, x_{2} p_{1}, x_{2} p_{2}, x_{1} p_{1}+x_{2} p_{2} \\
& p_{1}, p_{2}, x_{2} p_{2}+p_{3}, x_{1} p_{1}+\dot{\epsilon}^{x_{3}} p_{2}+x_{4} p_{3} \\
& p_{1}, p_{2}, x_{2} p_{2}, x_{1} p_{1}+p_{3} \\
& p_{1}, p_{2}, x_{2} p_{2}+p_{3}, x_{1} p_{1}+\epsilon^{x_{3}} p_{2}+c p_{3} \\
& p_{1}, x_{2} p_{1}, x_{2} p_{2}, x_{1} p_{1}+x_{2} p_{2}+p_{3} \\
& p_{1}, p_{2}, x_{3} p_{1}+x_{2} p_{2}, x_{1} p_{1}+x_{3} p_{3} \\
& p_{1}, p_{2}, x_{2} p_{2}+p_{3}, x_{1} p_{1}+\epsilon^{x_{3}} p_{2}+p_{4}
\end{aligned}
$$


THE $G_{r}(r<5)$ OF $n$-DIMENSIONAL SPACE.

31

III. $\left(U_{1} U_{2}\right)=\left(U_{1} U_{3}\right)=0, \quad\left(U_{2} U_{3}\right)=U_{1}, \quad\left(U_{1} U_{4}\right)=c U_{1}$,

$$
\left(U_{2} U_{4}\right)=U_{2}, \quad\left(U_{3} U_{4}\right)=(c-1) U_{3} \quad(c \neq 1) .
$$$$
p_{1}, p_{2}, x_{2} p_{1}, c x_{1} p_{1}+x_{2} p_{2}
$$$$
p_{1}, p_{2}, x_{2} p_{1}, x_{3} p_{1}+x_{2} p_{2}
$$$$
p_{1}, p_{2}, x_{2} p_{1}+x_{3} p_{2}, 2 x_{1} p_{1}+x_{2} \dot{p}_{2}
$$$$
p_{1}, p_{2}, x_{2} p_{1}+p_{3}, c x_{1} p_{1}+x_{2} p_{2}+(c-1) x_{3} p_{3}
$$$$
p_{1}, x_{2} p_{1}, p_{2}, c x_{1} p_{1}+(c-1) x_{2} p_{2}
$$$$
p_{1}, x_{2} p_{1}, p_{2}, x_{3} p_{1}-x_{2} p_{2}
$$$$
(c=0) \text {. }
$$$$
p_{1}, p_{2}, x_{2} p_{1}, c x_{1} p_{1}+x_{2} p_{2}+p_{3}
$$$$
p_{1}, p_{2}, x_{2} p_{1}+x_{3} p_{2}, c x_{1} p_{1}+x_{2} p_{2}+(2-c) x_{3} p_{3}
$$$$
p_{1}, p_{2}, x_{2} p_{1}+x_{3} p_{2}, 2 x_{1} p_{1}+x_{2} p_{2}+p_{4}
$$$$
p_{1}, p_{2}, x_{2} p_{1}+p_{3}, c x_{1} p_{1}+x_{2} p_{2}+(c-1) x_{3} p_{3}+p_{1}
$$$$
p_{1}, x_{2} p_{1}, p_{2}, c x_{1} p_{1}+(c-1) x_{2} p_{2}+p_{3}
$$$$
p_{1}, x_{2} p_{1}, p_{2}, p_{1}-x_{2} p_{2}
$$

$$
\begin{aligned}
& \text { IV. }\left(U_{1} U_{2}\right)=\left(U_{1} U_{3}\right)=0, \quad\left(U_{2} U_{3}\right)=U_{1}, \quad\left(U_{1} U_{1}\right)=2 U_{1} \text {, } \\
& \left(U_{2} U_{4}\right)=U_{2}, \quad\left(U_{3} U_{4}\right)=U_{2}+U_{3} \text {. } \\
& p_{1}, x_{2} p_{1}, p_{2},\left(2 x_{1}-\frac{1}{2} x_{2}^{2}\right) p_{1}+x_{2} p_{2} \\
& p_{1}, p_{2}, x_{2} p_{1}+p_{3},\left(2 x_{1}+\frac{1}{2} x_{3}^{2}\right) p_{1}+\left(x_{2}+x_{3}\right) p_{2}+\left(x_{3}+x_{1}\right) p_{3} \\
& p_{1}, x_{2} p_{1}, p_{2},\left(2 x_{1}-\frac{1}{2} x_{2}^{2}\right) p_{1}+x_{2} p_{2}+p_{3} \\
& p_{1}, p_{2}, x_{2} p_{1}+x_{3} p_{2}, 2 x_{1} p_{1}+x_{2} p_{2}-p_{3}
\end{aligned}
$$


32

'WILLIAMS B. STONE.

$$
p_{1}, p_{2}, x_{2} p_{1}+p_{3},\left(2 x_{1}+\frac{1}{2} x_{3}^{2}\right) p_{1}+\left(x_{2}+x_{3}\right) p_{2}+x_{3} p_{3}+p_{4}
$$

V. $\left(U_{1} U_{2}\right)=\left(U_{1} U_{3}\right)=0, \quad\left(U_{2} U_{3}\right)=U_{1}, \quad\left(U_{1} U_{4}\right)=U_{1}$, $\left(U_{2} U_{4}\right)=U_{2}, \quad\left(U_{3} U_{4}\right)=0$.

$$
p_{1}, p_{2}, x_{2} p_{1}, x_{1} p_{1}+x_{2} p_{2} \quad p_{1}, x_{2} p_{1}, p_{2}, x_{1} p_{1}
$$

$$
p_{1}, p_{2}, x_{2} p_{1}+p_{3}, x_{1} p_{1}+x_{2} p_{2}+F\left(x_{4}, \cdots, x_{n}\right) p_{3}
$$

$$
p_{1}, p_{2}, x_{2} p_{1}, x_{1} p_{1}+x_{2} p_{2}+p_{3} \quad p_{1}, x_{2} p_{1}, p_{2}, x_{1} p_{1}+p_{3}
$$$$
p_{1}, p_{2}, x_{2} p_{1}+x_{3} p_{2}, x_{1} p_{1}+x_{2} p_{2}+x_{3} p_{3}
$$$$
p_{1}, p_{2}, x_{2} p_{1}+p_{3}, x_{1} p_{1}+x_{2} p_{2}+p_{4}
$$

VI. $\left(U_{1} U_{4}\right)=U_{1}, \quad\left(U_{2} U_{4}\right)=a U_{2}, \quad\left(U_{3} U_{4}\right)=c U_{3}$,

$$
\begin{aligned}
& \left(U_{i} U_{k}\right)=0 \\
& (i, k=1,2,3) \\
& p_{1}, x_{2} p_{1}, F\left(x_{2}, \cdots, x_{n}\right) p_{1}, x_{1} p_{1} \\
& (a=c=1) \\
& p_{1}, p_{2}, x_{3} p_{2}, x_{1} p_{1}+a x_{2} p_{2} \\
& (a=c) \\
& p_{1}, x_{3} p_{1}, p_{2}, x_{1} p_{1}+c x_{2} p_{2} \\
& (a=1) \\
& p_{1}, p_{2}, x_{3} p_{1}+F\left(x_{3}, \cdots, x_{n}\right) p_{2}, x_{1} p_{1}+x_{2} p_{2} \quad(a=0=1) \\
& p_{1}, p_{2}, p_{3}, x_{1} p_{1}+a x_{2} p_{2}+c x_{3} p_{3} \\
& p_{1}, x_{2} p_{1}, F_{1}\left(x_{2}, \cdots, x_{n}\right) p_{1}, x_{1} p_{1}+F_{2}\left(x_{2}, \cdots, x_{n}\right) p_{4} \quad(a=1) \\
& p_{1}, x_{2} p_{1}, x_{3} p_{1}, p_{1}+(1-c) x_{3} p_{3} \\
& (a=1) \\
& p_{1}, x_{2} p_{1}, x_{3} p_{1}, p_{1}+(1-a) x_{2} p_{2}+(1-c) x_{3} p_{3} \\
& p_{1}, x_{2} p_{1}, x_{2}^{(1-o)(1-a)} p_{1}, x_{1} p_{1}+(1-a) x_{2} p_{2} \\
& p_{1}, p_{2}, x_{3} p_{2}, x_{1} p_{1}+\alpha x_{2} p_{2}+p_{4} \\
& (a=0)
\end{aligned}
$$


THE $G_{r}(r<5)$ OF $n$-DIMENGIONAL SPACE.

$p_{1}, p_{2}, x_{3} p_{2}, x_{1} p_{1}+a x_{2} p_{2}+F\left(x_{3}\right) p_{3}$

$p_{1}, x_{3} p_{1}, p_{2}, x_{1} p_{1}+c x_{2} p_{2}+(1-a) x_{3} p_{3}$

$p_{1}, p_{2}, x_{3} p_{1}+x_{4} p_{2}, x_{1} p_{1}+a x_{2} p_{2}+(1-a) x_{4} p_{4}$

$p_{1}, p_{2}, x_{3} p_{1}+F_{1}\left(x_{3}, \cdots, x_{n}\right) p_{2}, x_{1} p_{1}+x_{2} p_{2}+F_{2}\left(x_{3}, \cdots, x_{n}\right) p_{b}$

$(a=c=1)$

$p_{1}, p_{2}, x_{3} p_{1}+x_{4} p_{2}, x_{1} p_{1}+a x_{2} p_{2}+(1-c) x_{3} p_{3}+(a-c) x_{4} p_{4}$

$p_{1}, p_{2}, p_{3}, x_{1} p_{1}+a x_{2} p_{2}+c x_{3} p_{3}+p_{1}$

VII. $\left(U_{1} U_{1}\right) \equiv c U_{1}, \quad\left(U_{2} U_{4}\right) \equiv(1+c) U_{2}, \quad\left(U_{3} U_{4}\right) \equiv U_{1}+c U_{3}$,

$$
\left(U_{i} U_{k}\right)=0 \quad(i, k,=1,2,3) .
$$

$p_{1}, p_{2}, p_{3},\left(c x_{1}+x_{3}\right) p_{1}+(1+c) x_{2} p_{2}+c x_{3} p_{3} \quad(c \neq 0)$

$p_{1}, p_{2}, p_{3}, x_{3} p_{1}+x_{2} p_{2}+F\left(x_{1}, \cdots, x_{n}\right) p_{3} \quad(c=0)$

$p_{1}, x_{2} p_{1}, x_{3} p_{1}, c x_{1} p_{1}-x_{2} p_{2}-p_{3}$

$p_{1}, x_{2} p_{1}, \log x_{2} p_{1}, c x_{1} p_{1}-x_{2} p_{2}$

$p_{1}, x_{3} p_{1}, p_{2},\left(c x_{1}+x_{2}\right) p_{1}+c x_{2} p_{2}+x_{3} p_{3}$

$p_{1}, p_{2}, x_{3} p_{1}+x_{4} p_{2}, c x_{1} p_{1}+(1+c) x_{2} p_{2}-p_{3}+x_{4} p_{4}$

$p_{1}, p_{2}, x_{3} p_{1}+\epsilon^{-x_{3}} p_{2}, c x_{1} p_{1}+(1+c) x_{2} p_{2}-p_{3}$

$p_{1}, p_{2}, p_{3},\left(\mathrm{cx} x_{1}+x_{3}\right) p_{1}+(1+c) x_{2} p_{2}+c x_{3} p_{3}+p_{4}$

VIII. $\left(U_{1} U_{1}\right)=U_{2} f, \quad\left(U_{2} U_{1}\right)=0, \quad\left(U_{3} U_{1}\right)=U_{1}$,

$$
\left(U_{i} U_{k}\right)=0 \quad(i, k,=1,2,3) .
$$

$p_{1}, p_{2}, p_{3}, x_{3} p_{1}+x_{1} p_{2}+F\left(x_{1}, \cdots, x_{n}\right) p_{3}$

$p_{1}, x_{2} p_{1}, x_{3} p_{1}, x_{1} x_{2} p_{1}+x_{2}^{2} p_{2}+\left(x_{2} x_{3}-1\right) p_{3}$ 


$$
\begin{aligned}
& p_{1}, x_{2} p_{1}, x_{2}^{-1} p_{1}, x_{1} x_{2} p_{1}+x_{2}^{2} p_{2} \\
& p_{1}, x_{3} p_{1}, p_{2},\left(x_{1} x_{3}+x_{2}\right) p_{1}+x_{3}^{2} p_{3} \\
& p_{1}, p_{2}, x_{3} p_{1}+x_{4} p_{2}, x_{1} p_{2}-p_{3}+x_{3} p_{4} \\
& p_{1}, p_{2}, 2 x_{3} p_{1}-x_{3}^{2} p_{2}, x_{1} p_{2}-p_{3} \\
& p_{1}, p_{2}, p_{3}, x_{3} p_{1}+x_{1} p_{2}+p_{4} \\
& \text { IX. }\left(U_{1} U_{4}\right)=U_{1} f+U_{2} f,\left(U_{2} U_{4}\right)=U_{2} f,\left(U_{3} U_{4}\right)=U_{1} f+U_{3} f \text {, } \\
& \left(U_{i}, U_{k}\right)=0 \quad(i, k=1,2,3) . \\
& p_{1}, p_{2}, p_{3},\left(x_{1}+x_{3}\right) p_{1}+\left(x_{1}+x_{2}\right) p_{2}+x_{3} p_{3} \\
& p_{1}, x_{2} p_{1}, x_{3} p_{1},\left(x_{1}+x_{1} x_{2}\right) p_{1}+x_{2}^{2} p_{2}+\left(x_{2} x_{3}-1\right) p_{3} \\
& p_{1}, x_{2} p_{1}, x_{2}^{-1} p_{1},\left(x_{1}+x_{1} x_{2}\right) p_{1}+x_{2}^{2} p_{2} \\
& p_{1}, x_{3} p_{1}, p_{2},\left(x_{1}+x_{1} x_{3}+x_{2}\right) p_{1}+x_{2} p_{2}+x_{3}^{2} p_{3} \\
& p_{1}, p_{2}, x_{3} p_{1}+x_{1} p_{2}, x_{1} p_{1}+\left(x_{1}+x_{2}\right) p_{2}-p_{3}+x_{3} p_{4} \\
& p_{1}, p_{2}, 2 x_{3} p_{1}-x_{3}^{2} p_{2}, x_{1} p_{1}+\left(x_{1}+x_{2}\right) p_{2}-p_{3} \\
& p_{1}, p_{2}, p_{3},\left(x_{1}+x_{3}\right) p_{1}+\left(x_{1}+x_{2}\right) p_{2}+x_{3} p_{3}+p_{1} \\
& \text { X. }\left(U_{3} U_{4}\right)=U_{2} f,\left(U_{1} U_{t}\right)=\left(U_{2} U_{4}\right)=\left(U_{i}, U_{k}\right)=0 \quad(i, k=1,2,3) \\
& p_{1}, x_{3} p_{1}, p_{2}, x_{2} x_{3} p_{1}+F\left(x_{3}, \cdots, x_{n}\right) p_{2} \\
& p_{1}, p_{2}, p_{3}, x_{4} p_{1}+x_{3} p_{2}+F\left(x_{4}, \cdots, x_{n}\right) p_{3} \\
& p_{1}, x_{2} p_{1}, x_{3} p_{1}, x_{2} p_{3} \\
& p_{1}, p_{2}, x_{3} p_{2}, p_{3} \\
& p_{1}, x_{3} p_{1}, p_{2}, x_{2} x_{3} p_{1}+F\left(x_{3}, \cdots, x_{n}\right) p_{4}
\end{aligned}
$$


THE $G_{r}(r<5)$ OF $n$-DTMENSIONAL SPAOE.

$$
p_{1}, p_{2}, x_{3} p_{1}+x_{4} p_{2}, p_{4}
$$

$$
p_{1}, p_{2}, p_{3}, x_{3} p_{2}+p_{1}
$$

XI. $\left(U_{1} U_{1}\right)=U_{1} f, \quad\left(U_{2} U_{1}\right)=U_{2} f, \quad\left(U_{3} U_{1}\right)=U_{2} f+U_{3} f$,

$$
\left(U_{i} U_{k}\right)=0 \quad(i, k=1,2,3) \text {. }
$$

$p_{1}, x_{3} p_{1}, p_{2},\left(x_{1}+x_{2} x_{3}\right) p_{1}+x_{2} p_{2}$

$p_{1}, p_{2}, p_{3}, x_{1} p_{1}+\left(x_{2}+x_{3}\right) p_{2}+x_{3} p_{3}$

$p_{1}, x_{2} p_{1}, x_{3} p_{1}, x_{1} p_{1}-x_{2} p_{3}$

$p_{1}, p_{2}, x_{3} p_{2}, x_{1} p_{1}+x_{2} p_{2}-p_{3}$

$p_{1}, x_{3} p_{1}, p_{2},\left(x_{1}+x_{2} x_{3}\right) p_{1}+x_{2} p_{2}+F\left(x_{3}, \cdots, x_{n}\right) p_{4}$

$p_{1}, p_{2}, x_{3} p_{1}+x_{4} p_{2}, x_{1} p_{1}+x_{2} p_{2}-p_{4}$

$p_{1}, p_{2}, p_{3}, x_{1} p_{1}+\left(x_{2}+x_{3}\right) p_{2}+x_{3} p_{3}+p_{4}$

XII.

$$
\left(U_{i}, U_{k}\right)=0 \quad(i, k=1,2,3,4) .
$$

$p_{1}, x_{2} p_{1}, F_{1}\left(x_{2}, \cdots, x_{n}\right) p_{1}, F_{2}\left(x_{2}, \cdots, \dot{x}_{n}\right) p_{1}$

$p_{1}, p_{2}, x_{3} p_{2}, F_{1}\left(x_{3}, \cdots, x_{n}\right) p_{1}+F_{2}\left(x_{3}, \cdots, x_{n}\right) p_{2}$

$p_{1}, p_{2}, x_{3} p_{1}+F_{1}\left(x_{3}, \cdots, x_{n}\right) p_{2}, F_{2}\left(x_{3}, \cdots, x_{n}\right) p_{1}+F_{3}\left(x_{3}, \cdots, x_{n}\right) p_{2}$

$$
p_{1}, p_{2}, p_{3}, x_{4} p_{1}+x_{5} p_{2}+x_{6} p_{3}
$$

$p_{1}, x_{2} p_{1}, x_{3} p_{1}, p_{4} \quad p_{1}, x_{2} p_{1}, F_{1}\left(x_{2}\right) p_{1}, F_{2}\left(x_{2}, \cdots, x_{n}\right) p_{3}$

$$
p_{1}, p_{2}, x_{3} p_{2}, F\left(x_{3}, \cdots, x_{n}\right) p_{4}
$$

$p_{1}, p_{2}, x_{3} p_{1}+x_{4} p_{2}, F\left(x_{3}, \cdots, x_{n}\right) p_{j} \quad(j>4)$

$p_{1}, p_{2}, x_{3} p_{1}+F_{1}\left(x_{3}\right) p_{2}, F_{2}\left(x_{3}, \cdots, x_{n}\right) p_{j} \quad(j>3)$

$p_{1}, p_{2}, p_{3}, p_{4}$ 


\section{CHAPTER II.}

\section{INVARIANTS OF THE $G_{r}(r<4)$.}

Having established all the groups of two, three, and four parameters in $n$ variables, we are next concerned with the finding of their differential invariants when $n<4$. In this problem a division is to be made according as $y$ and $z$ are functions of $x$; or $z$ is a function of $x$ and $y$. The calculations for finding the invariants for these two cases are different and will be given in different sections.

\section{\$1. Invariants When $y$ and $z$ are Functions of $x$.}

In this section we shall give the detailed work for several of the more difficult types, and tabulate the remaining results at the end of the paper- Lie's general method consists in equating to zero the transformations after they have been extended, and finding the solutions of the resulting complete systems.

As is customary, we shall use $y_{1}, z_{1}$ to represent the total first derivatives of $y$ and $z$ respectively with respect to $x ; y_{2}, z_{2}$, the total second derivatives, etc. The expressions by means of which the increments of the higher derivatives are obtained were deduced by $\mathrm{Lie}$, and for this case they have the forms

$$
\eta_{(n)} \equiv \frac{d \eta_{(n-1)}}{d x}-y_{n} \frac{d \xi}{d x}, \quad \zeta_{(n)} \equiv \frac{d \zeta_{(n-1)}}{d x}-z_{n} \frac{d \xi}{d x}
$$

where $\eta_{(n)}, \zeta_{(n)}$ represent the increments, which $y_{n}, z_{n}$, respectively receive.

1. Let us find first the differential invariants of the group,

$$
p, x p+y q, x^{2} p+2 x y q \text {. }
$$

Calculating the increments of $y_{1}, z_{1}$ for each of the transformations by means of the expressions given above, we have for the onceextended transformations : 
THE $G_{r}(r<5)$ OF $n$-DIMENSIONAL SPAOE.

$$
\begin{aligned}
& U_{1}^{\prime} f \equiv p=0 \\
& U_{2}^{\prime} f \equiv x p+y q+0 r+0 q_{1}-z_{1} r_{1}=0 \\
& U_{3}^{\prime} f=x^{2} p+2 x y q+0 r+2 y q_{1}-2 x z_{1} r_{1}=0 \\
& \quad\left(q_{1} \equiv \frac{\partial f}{\partial y_{1}}, \quad r_{1} \equiv \frac{\partial f}{\partial z_{1}}, \quad \text { etc. }\right) .
\end{aligned}
$$

The first equation shows that $x$ does not occur in any of the invariants.

The second linear partial differential equation is equivalent to

$$
\frac{d y}{y}=\frac{d z}{0}=\frac{d y_{1}}{0}=-\frac{d z_{1}}{z_{1}}
$$

of which the integrals are $z, y_{1}, y z_{1} \equiv u$.

Introducing these as new variables into $U_{3}^{\prime} f=0$, the coefficients in the new equation can be expressed as functions of the new variables. Performing the operation, we get

$$
\bar{U}_{3}^{\prime} f \equiv 0 r+q_{1}+0 \frac{\partial f}{\partial u}=0 \text {. }
$$

The solutions of this linear partial differential equation are directly $z, u \equiv y z_{1}$.

To find the invariants of the second order, the transformations must be extended twice. They then have the forms :

$U_{1}^{\prime \prime} f \equiv p=0$,

$U_{2}^{\prime \prime} f \equiv x p+y q+0 r+0 q_{1}-z_{1} r_{1}-y_{2} q_{2}-2 z_{2} r_{2}=0$,

$U_{3}^{\prime \prime} f \equiv x^{2} p+2 x y q+0 r+2 y q_{1}-2 x z_{1} r_{1}$

$$
+2\left(y_{1}-x y_{2}\right) q_{2}-2\left(z_{1}+2 x z_{2}\right) r_{2}=0 \text {. }
$$

The third equation can be considerably simplified by replacing it by $\nabla_{3}^{\prime \prime} f=U_{3}^{\prime \prime} f-2 x U_{2}^{\prime \prime} f+x^{2} U_{1}^{\prime \prime} f$, giving

$$
V_{3}^{\prime \prime} f \equiv 0 r+2 y q_{1}+2 y_{1} q_{2}-2 z_{1} r_{2}=0 \text {. }
$$

As before, $U_{1}^{\prime \prime} f$ merely shows that the invariants are independent of $x$. Then the solutions of $U_{2}^{\prime \prime} f$ are directly

$$
z, y_{1}, y z_{1} \equiv u, y y_{2} \equiv v, y^{2} z_{2} \equiv w \text {. }
$$

Introducing these solutions as new variables into $\nabla_{3}^{\prime \prime} f$, it becomes

$$
\bar{V}_{3}^{\prime \prime} f=0 \frac{\partial f}{\partial z}+\frac{\partial f}{\partial y_{1}}+0 \frac{\partial f}{\partial u}+2 y_{1} \frac{\partial f}{\partial v}-u \frac{\partial f}{\partial w},
$$


WILLIAN B. STONE.

of which the solutions are: $z, u, y_{1}^{2}-2 v, y_{1}+w / u$. Hence the invariants of the second order are

$$
y_{1}^{2}-2 y y_{2}, \quad y_{1}+\frac{y z_{2}}{z_{1}}
$$

By continuing to extend the transformations in this way, the differential invariants of any order can be found; but the work becomes very complicated after a few extensions and a simpler method is at hand.

This method makes use of the differential parameter to find the invariants of higher orders. If $\phi$ is an invariant of lowest order, we seek a function

$$
\Omega\left(x, y, z, y_{1}, z_{1}, \cdots, \phi, \frac{d \phi}{d x}\right)
$$

which shall be a differential invariant whenever $\phi$ is.

Indicating by $\delta$ the increment received by means of an infinitesimal transformation of the group, we have, since $d \phi \equiv \phi^{\prime} d x$,

$$
\therefore \quad \cdot \delta d \phi=\delta \phi^{\prime} d x+\phi^{\prime} \delta d x
$$

or since the operations denoted by $d$ and $\delta$ are interchangeable,

$$
\delta \phi^{\prime} \equiv \frac{d \delta \phi}{d x}-\phi^{\prime} \frac{d \delta x}{d x}
$$

Now if $\phi$ is an invariant, it receives no increment, i. e., $\delta \phi=0$, so that

$$
\delta \phi^{\prime} \equiv-\phi^{\prime} \frac{d \delta x}{d x}
$$

This is the expression to determine the increment, which $\phi^{\prime}$ receives by means of a transformation.

Applying to the transformations of the group under consideration, we have the following complete system:

$$
\begin{aligned}
& U_{1} f \equiv \frac{\partial \Omega}{\partial x}=0 \\
& U_{2} f \equiv x \frac{\partial \Omega}{\partial x}+y \frac{\partial \Omega}{\partial y}-\phi^{\prime} \frac{\partial \Omega}{\partial \phi^{\prime}}=0 \\
& U_{3} f \equiv x^{2} \frac{\partial \Omega}{\partial x}+2 x y \frac{\partial \Omega}{\partial y}-2 x \phi^{\prime} \frac{\partial \Omega}{\partial \phi^{\prime}}=0 .
\end{aligned}
$$


THE $G_{r}(r<5)$ OF $n$-DIMENSIONAL SPACE.

One common solution of this system is found immediately to be $y \phi^{\prime}$. Hence if $\phi$ is an invariant under the transformations of the group $\Delta \phi \equiv y \phi^{\prime}$, obtained by multiplying the first derivative of $\phi$ with respect to $x$ by $y$, is also an invariant.

Thus, knowing the two invariants above of second order, we can by this process find those of the third order; then applying the parameter to them, find those of the fourth order, and so on.

(2) Let us consider next the group

$$
p, q, y p+z q \text {. }
$$

The invariants are obtained more easily if, by the introduction of the new variables

$$
\overline{\mathrm{x}}=\frac{x}{z}, \quad \bar{y}=\frac{y}{z}, \quad \bar{z}=\frac{1}{z},
$$

we change the group into the similar one

$$
\therefore z p, z q, y p+q
$$

The twice-extended forms of these last transformations are:

$U_{1}^{\prime \prime} f \equiv z p-y_{1} z_{1} q_{1}-z_{1}^{2} r_{1}-\left(y_{1} z_{2}+2 y_{2} z_{1}\right) q_{2}-3 z_{1} z_{2} r_{2}=0$,

$U_{2}^{\prime \prime} f \equiv z q+z_{1} q_{1}+z_{2} q_{2}=0$,

$U_{3}^{\prime \prime} f \equiv y p+q-y_{1}^{2} q_{1}-y_{1} z_{1} r_{1}-3 y_{1} y_{2} q_{2}-\left(2 y_{1} z_{2}+y_{2} z_{1}\right) r_{2}=0$.

The solutions of the second equation are

$$
x, z, z_{1}, z_{2}, z y_{1}-z_{1} y \equiv u, z y_{2}-z_{2} y \equiv v .
$$

Introducing these as new variables into the first equation, it becomes

$$
\bar{U}_{1}^{\prime \prime} f \equiv z p+0 r-z_{1}^{2} r_{1}-3 z_{1} z_{2} r_{2}-u z_{2} \frac{\partial f}{\partial u}-\left(u z_{2}+2 v z_{1}\right) \frac{\partial f}{\partial v}=0 \text {. }
$$

The integrals of the corresponding simultaneous system are

$$
z, \frac{x}{z}-\frac{1}{z_{1}} \equiv \rho_{1}, \frac{z_{1}^{3}}{z_{2}} \equiv \rho_{2}, \quad \frac{u}{z_{1}} \equiv \rho_{3}, \frac{v}{z_{1}^{2}}-\frac{u z_{2}}{z_{1}^{3}} \equiv \rho_{4} .
$$

Introducing these as new variables into the third equation, we have

$$
\bar{U}_{3}^{\prime \prime} f \equiv 0 r-\frac{\rho_{3}}{z} \frac{\partial f}{\partial \rho_{1}}+\frac{\rho_{1} \rho_{2}^{2}}{z} \cdot \frac{\partial f}{\partial \rho_{2}}-\frac{\partial f}{\partial \rho_{3}}+0 \frac{\partial f}{\partial \rho_{4}}=0
$$


of which the solutions are

or in the original variables

$$
z, 2 z \rho_{1}-\rho_{3}^{2}, \rho_{4}, \frac{z}{\rho_{4} \rho_{2}}-\rho_{3}
$$

$$
\begin{gathered}
z, 2 x-\frac{2 z}{z_{1}}-\frac{\left(z y_{1}-z_{1} y\right)^{2}}{z_{1}^{2}}=I_{1} ; \quad \frac{z\left(z_{1} y_{2}-z_{2} y_{1}\right)}{z_{1}^{3}}=I_{2} ; \\
\frac{z_{2}}{z_{1} y_{2}-z_{2} y_{1}}-\frac{z y_{1}-z_{1} y}{z_{1}} \equiv J_{2} .
\end{gathered}
$$

By the method of the preceding paragraph, we find

$$
\Delta \phi \equiv \frac{\phi^{\prime}}{z_{1}} \text {. }
$$

Applying this to the invariants $I_{2}, J_{2}$, above, we find $I_{3}, J_{3}$ of the third order, etc.

We will now tabulate all the groups of two and three parameters and their total differential invariants. The classification is made according to the order of the first derived groups. The invariants are found up to the second order and we will use $I_{1}, . J_{1}$, to represent those of the first order, $I_{2}, J_{2}$, those of the second order. The invariants of higher orders can easily be obtained by the use of the differential parameter for each group, as explained on pages 3839. From these invariants, the invariant differential equations can easily be set up by equating an arbitrary function of the invariants to zero.

$$
G_{2}^{\prime} \text { '. }
$$

I. When the first-derived group is one-membered :

$$
\begin{aligned}
& {[1] \quad p, x p: I_{1} \equiv \frac{z_{1}}{y_{1}} ; \quad I_{2} \equiv \frac{y_{2}}{y_{1}^{2}} ; \quad J_{2} \equiv \frac{z_{2}}{z_{1}^{2}} ; \quad \Delta \phi \equiv \frac{\phi^{\prime}}{z_{1}} .} \\
& {[2]: I_{1} \equiv y_{1} ; \quad J_{1} \equiv z_{1} ; \quad I_{2} \equiv z y_{2} ; \quad J_{2} \equiv z z_{2} ;} \\
& \Delta \phi \equiv z \phi^{\prime} .
\end{aligned}
$$

II. When the first-derived group is zero-membered :

$$
\begin{aligned}
& p, z p: \quad I_{1} \equiv \frac{y_{1}}{z_{1}} ; \quad I_{2} \equiv \frac{z_{2}}{z_{1}^{3}} ; \quad J_{2} \equiv \frac{y_{1} z_{2}-y_{2} z_{1}}{z_{1}^{3}} ; \quad \Delta \phi \equiv \frac{\phi^{\prime}}{z_{1}} \\
& p, q: I_{1} \equiv y_{1} ; \quad J_{1} \equiv z_{1} ; \quad I_{2} \equiv y_{2} ; \quad J_{2} \equiv z_{2} ; \quad \Delta \phi \equiv \phi^{\prime} .
\end{aligned}
$$


THE $G_{r}(r<5)$ OF $n$-DIMENSTONAL SPAOE.

$G_{3}^{\prime}$ 's.

I. When the first-derived group is three-membered.

[1]

$p, x p, x^{2} p, I_{1} \equiv \frac{y_{1}}{z_{1}} ; \quad I_{2} \equiv \frac{y_{1} z_{2}-y_{2} z_{1}}{y_{1}^{3}} ; \quad \Delta \phi \equiv \frac{\phi^{\prime}}{z_{1}}$.

[2]

$\left.p, x p+y q, x^{2} p+2 x y q\right]: I_{1} \equiv y z_{1} ; \quad I_{2} \equiv y_{1}^{2}-2 y y_{2} ;$

$J_{2} \equiv \frac{y_{1} z_{1}+y z_{2}}{z_{1}} ; \quad \Delta \phi \equiv y \phi^{\prime}$.

[3]

$p, x p+y q, x^{2} p+\left(2 x y+y^{2}\right) q: I_{1} \equiv \frac{y^{2} z_{1}^{2}}{1+y_{1}}$

$I_{2} \equiv y^{2} z_{2}-2 y z_{1} ; \quad J_{2} \equiv y y_{2}-\left(1+y_{1}\right)^{2} \int \frac{y_{1} d y_{1}}{\left(1+y_{1}\right)^{1}} ;$

$\Delta \phi \equiv \frac{\phi^{\prime}}{z_{1}}$.

[4]

$p, x p+y q, x^{2} p+2 x y q+y r: I_{1} \equiv 2 z-y_{1} ; J_{1} \equiv y_{1}^{2}-4 y z_{1} ;$

$I_{2} \equiv y y_{2}-2 y z_{1} ; J_{2} \equiv y z\left(y_{2}-2 z_{1}\right)-y^{2} z_{2} ; \Delta \phi \equiv y \phi^{\prime}$.

II. When the first-dorived group is two-membered:

[5] $r, x r, z r: I_{1} \equiv y_{1} ; \quad I_{2} \equiv y_{2} ; \quad \Delta \phi \equiv \phi^{\prime}$.

[6] $r, x r, q+z r: I_{1} \equiv y_{1} ; \quad I_{2} \equiv y_{2} ; J_{2} \equiv \epsilon^{-y} \cdot z_{2} ; \quad \Delta \phi \equiv \phi^{\prime}$.

[7] $r, x r,(1-c) x p+z r \mid I_{1} \equiv x y_{1} ; I_{2} \equiv x^{2} y_{2} ; J_{2} \equiv x^{1-2 c} \cdot z_{2}^{1-c}$;

$$
\Delta \phi \equiv \frac{\phi^{\prime}}{y_{1}} \text {. }
$$

[8] $p, q, x p+z q: I_{1} \equiv \frac{y_{1}}{z_{1}}+\log z_{1} ; \quad I_{2} \equiv z_{1}^{-2} \cdot z_{2}$;

$$
J_{2} \equiv \frac{2 y_{2}}{z_{2}}+\log z_{2} ; \quad \Delta \phi \equiv \frac{\phi^{\prime}}{z_{1}}
$$

[9]

$p, q, x p+c y q ; I_{1} \equiv y_{1} z_{1}^{c-1} ; \quad I_{2} \equiv y_{1}^{c-2} \cdot y_{2}^{1-c} ; \quad J_{2} \equiv z_{1}^{-2} z_{2} ;$

$$
\Delta \phi \equiv \frac{\phi^{\prime}}{z_{1}}
$$


$p, q, x p+c y q+r: I_{1} \equiv z+\log z_{1} ; \quad J_{1} \equiv z_{1} \cdot y_{1}^{1 /(c-1)}$

$$
I_{2} \equiv z_{1}^{c-2} y_{2} ; \quad J_{2} \equiv z_{1}^{-2} z_{2} ; \quad \Delta \phi \equiv \frac{\phi^{\prime}}{z_{1}} .
$$

[11]

$r, x r, p-z r \cdot I_{1} \equiv y_{1} ; \quad I_{2} \equiv y_{2} ; \quad J_{2} \equiv \epsilon^{x} \cdot z_{2} ; \quad \Delta \phi \equiv \phi^{\prime}$

[12]

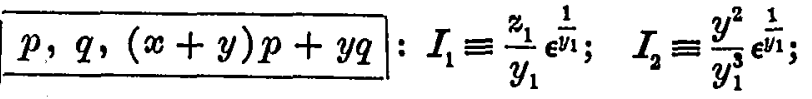

$$
\begin{aligned}
& J_{2} \equiv \epsilon^{\frac{2}{y_{1}}}\left(\frac{y_{1} z_{2}-y_{2} z_{1}}{y_{1}^{3}}\right) ; \Delta \phi \equiv \frac{\phi^{\prime}}{z_{1}} . \\
& p, q,(x+y) p+y q+r: I_{1} \equiv z-\frac{1}{y_{1}} ; \quad J_{1} \equiv \frac{z_{1}}{y_{1}} e^{\frac{1}{y_{1}}} ; \\
& I_{2} \equiv \frac{y_{2}}{y_{1}^{3}} \epsilon^{\frac{1}{y_{1}}} ; \quad J_{2} \equiv \epsilon^{\frac{2}{y_{1}}}\left(\frac{y_{1} z_{2}-y_{2} z_{1}}{y_{1}^{3}}\right) ; \quad \Delta \phi \equiv \frac{\phi^{\prime}}{z_{1}} \text {. }
\end{aligned}
$$

[13]

III. When the first-derived group is one-membered :

[14]

$$
\begin{aligned}
& p, q, x p: I_{1} \equiv \frac{y_{1}}{z_{1}} ; \quad I_{2} \equiv \frac{y_{2}}{z_{1}^{2}} ; \quad J_{2} \equiv \frac{z_{2}}{z_{1}^{2}} ; \quad \Delta \phi \equiv \frac{\phi^{\prime}}{z_{1}} . \\
& p, q, y p+z q \equiv z p, z q, y p+q: I_{1} \equiv \frac{x}{z}-\frac{1}{z_{1}} ; \quad I_{2} \equiv \frac{z_{1}^{3}}{z_{2}} ; \\
& J_{1} \equiv \frac{y z_{1}-z y_{1}}{z_{1}} ; \quad J_{2} \equiv \frac{z\left(y_{1} z_{2}-y_{2} z_{1}\right)}{z_{1}^{3}} ; \quad \Delta \phi \equiv \frac{\phi^{\prime}}{z_{1}} \text {. }
\end{aligned}
$$

[15]

[16]

$$
p, q, y p=I_{1} \equiv \frac{z_{1}}{y_{1}} ; \quad I_{2} \equiv \frac{y_{1}^{3}}{y_{2}} ; \quad J_{2} \equiv \frac{y_{2} z_{1}-y_{1} z_{2}}{y_{1}^{3}} ; \quad \Delta \phi \equiv \frac{\phi^{\prime}}{z_{1}} \text {. }
$$

[17] $p, q, y p+v: I_{1} \equiv z-\frac{1}{y_{1}} ; \quad J_{1} \equiv \frac{z_{1}}{y_{1}} ; \quad I_{2} \equiv \frac{y_{1}^{3}}{y_{2}}$;

$$
J_{2} \equiv \frac{y_{1} z_{2}-y_{2} z_{1}}{y_{1}^{3}} ; \quad \Delta \phi \equiv \frac{\phi^{\prime}}{z_{1}} \text {. }
$$

$r, x r, F(x, y) r: I_{1} \equiv y_{1} ; \quad I_{2} \equiv y_{2} ; \quad \Delta \phi \equiv \phi^{\prime}$.

$$
p, q, z p: I_{1} \equiv \frac{y_{1}}{z_{1}} ; \quad I_{2} \equiv \frac{z_{2}}{z_{1}^{3}} ; \quad J_{2} \equiv \frac{y_{1} z_{2}-y_{2} z_{1}}{z_{1}^{3}} ; \quad \Delta \phi \equiv \frac{\phi^{\prime}}{z_{1}}
$$


THE $G_{r}(r<5)$ OF n-DIMENSTONAL SPACE.

[20]

$$
\begin{aligned}
& {[p, q, z p+\eta(z) q): I_{1} \equiv \frac{y_{1}-\eta^{\prime}(z)}{z_{1}} ; \quad I_{2} \equiv \frac{z_{2}}{z_{1}^{3}} ;} \\
& J_{2} \equiv \frac{y_{2} z_{1}-y_{1} z_{2}+\eta^{\prime}(z) \cdot z_{2}-\eta^{\prime \prime}(z) \cdot z_{1}^{2}}{z_{1}^{3}} ; \quad \Delta \phi \equiv \frac{\phi^{\prime}}{z_{1}} . \\
& p, q, r: I_{1} \equiv y_{1} ; \quad J_{1} \equiv z_{1} ; \quad I_{2} \equiv y_{2} ; \quad J_{2} \equiv z_{2} ; \quad \Delta \phi \equiv \phi^{\prime} .
\end{aligned}
$$

§ 2. The Invariants when $z$ is a Function of $x$ and $y$.

In this section the invariants when $z$ is a function of $x$ and $y$ are found for the groups of two and three parameters. For the fourmembered groups the results are not given, though they can be deduced, by a theorem due to Lie (see, "Continuierliche Gruppen," page 761), from the total invariants of the $G_{4}$ 's, which have been obtained by the writer, though not given in this paper.

For these invariants, we shall make use of the following relations to determine the increments of the partial derivatives:

$$
\begin{aligned}
& d \delta z=\delta p d x+\delta q d y+p d \delta x+q d \delta y, \\
& d \delta p=\delta v d x+\delta s d y+r d \delta x+s d \delta y, \\
& d \delta q=\delta s d x+\delta t d y+s d \delta x+t d \delta y,
\end{aligned}
$$

$p, q, r, s, t$ standing respectively for

$$
\frac{\partial z}{\partial x}, \frac{\partial z}{\partial y}, \frac{\partial^{2} z}{\partial x^{2}}, \frac{\partial^{2} z}{\partial x \cdot \partial y}, \frac{\partial^{2} z}{\partial y^{2}} \text {. }
$$

In the same way the corresponding expression for finding the differential parameters is

$$
d \delta \phi=\delta \phi_{x} \cdot d x+\delta \phi_{y} \cdot d y+\phi_{x} d \delta x+\phi_{y} \cdot d \delta y .
$$

I. Let us consider first the groups. of two parameters, which were found in Chapter I.

$$
\frac{\partial f}{\partial z}, x \frac{\partial f}{\partial x}+y \frac{\partial f}{\partial y}+z \frac{\partial f}{\partial z} \text {. }
$$

The twice extended forms of these transformations, when $z$ is a function of $x$ and $y$, are

$$
\frac{\partial f}{\partial z}=0
$$


$x \frac{\partial f}{\partial x}+y \frac{\partial f}{\partial y}+z \frac{\partial f}{\partial z}+0 \frac{\partial f}{\partial p}+0 \frac{\partial f}{\partial q}-r \frac{\partial f}{\partial r}-s \frac{\partial f}{\partial s}-t \frac{\partial f}{\partial t}=0$.

The first equation shows merely that the invariants do not contain $z$. Then the solutions of the second are directly

$p, q, x r, x s, x t$.

By using the above-given expression for the differential parameters, they are found in the same way to be

(2) For the group

$$
x \phi_{x}, x \phi_{y} \text {. }
$$

$$
\frac{\partial f}{\partial x}, z \frac{\partial f}{\partial x}
$$

the invariants are found in the same way to be

$$
\frac{q}{p}, \frac{r}{p^{3}}, \frac{p s-q r}{p^{3}}, \frac{p^{2} t-2 p q s+q^{2} r^{r}}{p^{3}}
$$

and the differential parameters are

(3) For

$$
\frac{\phi_{x}}{p}, \frac{q \phi_{x}-p \phi_{y}}{q}
$$

$$
\frac{\partial f}{\partial x}, \frac{\partial f}{\partial y}
$$

the invariants are $p, q, r, s, t$, and the differential parameters $\phi_{x}, \phi_{y}$.

(4) For

$$
\frac{\partial f}{\partial x}, x \frac{\partial f}{\partial x}
$$

the invariants are $q, t, r / p^{2}, s / p$ and the parameters $\phi_{y}, \phi_{x} / p$.

II. We will now give in detail the finding of the invariants for some of the $G_{3}^{\prime} \mathrm{s}$, and tabulate the results of the rest.

(1) Let us consider the group

$$
\frac{\partial f}{\partial x}, \frac{\partial f}{\partial y}, z \frac{\partial f}{\partial x}+\eta(z) \frac{\partial f}{\partial y} \text {. }
$$


THE $G_{r}(r<5)$ OF $n$-DIMENSIONAL SPACE.

The twice extended forms of these transformations are

$$
\begin{aligned}
& \frac{\partial f}{\partial x}=0 \\
& \frac{\partial f}{\partial y}=0
\end{aligned}
$$

$z \frac{\partial f}{\partial x}+\eta(z) \frac{\partial f}{\partial y}-\dot{p}\left(p+q \eta^{\prime}\right) \frac{\partial f}{\partial p}-q\left(p+q \eta^{\prime}\right) \frac{\partial f}{\partial q}-(3 p r+q r \eta$

$$
\begin{aligned}
&\left.+2 p s \eta^{\prime}+p^{2} q \eta^{\prime \prime}\right) \frac{\partial f}{\partial r}-\left(2 p s+2 q s \eta^{\prime}+p t \eta^{\prime}+p q^{2} \eta^{\prime \prime}+q r\right) \frac{\partial f}{\partial s} \\
&-\left(2 q s+p t+3 q t \eta^{\prime}+q^{3} \eta^{\prime \prime}\right) \frac{\partial f}{\partial t}=0 .
\end{aligned}
$$

The first two equations show merely that the invariants are free of $\ddot{x}$ and $y$. The simultaneous system corresponding to the third is then

$$
\begin{aligned}
\frac{d z}{0} & =\frac{d p}{p\left(p+q \eta^{\prime}\right)}=\frac{d q}{q\left(p+q \eta^{\prime}\right)}=\frac{d r}{3 p r+q r \eta^{\prime}+2 p s \eta^{\prime}+p^{2} q \eta^{\prime \prime}} \\
& =\frac{d s}{2 p s+2 q 8 \eta^{\prime}+p t \eta^{\prime}+p^{2} q \eta^{\prime \prime}+q r}=\frac{d t}{2 q s+p t+3 q t \eta^{\prime}+q^{3} \eta^{\prime \prime}} .
\end{aligned}
$$

Two integrals are immediately $z, p / q$, and to find the remaining, the differential parameters have to be used. They are found from the system

$$
\frac{d p}{p\left(p+q \eta^{\prime}\right)}=\frac{d q}{q\left(p+q \eta^{\prime}\right)}=\frac{d \phi_{x}}{p\left(\phi_{x}+\phi_{y} \cdot \eta^{\prime}\right)}=\frac{d \phi}{q\left(\phi_{x}+\phi_{y} \cdot \eta^{\prime}\right)^{\prime}}
$$

of which the integrals are $\left(q \phi_{x}-p \phi_{y}\right) / q,\left(\phi_{x}+\phi_{y} \cdot \eta^{\prime}\right) / p$. Operating with these on the solution $p / q$, two solutions of the second order are found to be

$$
\frac{q^{2} r-2 p q s+p^{2} t}{i q^{3}} \text { and } \frac{q r-p s+(q s-p t) \eta^{\prime}}{p q^{2}}
$$

Equating $p / q$ and these two solutions of the second order to constants, using the second ratio of $(A)$ with the fourth, or fifth, or sixth, and eliminating, by means of the solutions equated to constants, from the denominators the variables that do not occur in the numerators, we have left ordinary differential equations of the first order, which are linear in $r$, or $s$, or $t$, respectively. These can be integrated by quadratures and give three solutions of the second order, of which the two above are functions. 
2. We will next find the invariants of the group

$$
\frac{\partial f}{\partial x}, \frac{\partial f}{\partial y}, y \frac{\partial f}{\partial x}+z \frac{\partial f}{\partial y} \text {. }
$$

As in the last section, we will use the similar group, obtained by the introduction of the new variables

$$
\begin{aligned}
& \bar{x}=\frac{x}{z}, \quad \bar{y}=\frac{y}{z}, \quad \bar{z}=\frac{1}{z}, \\
& z \frac{\partial f}{\partial x}, z \frac{\partial f}{\partial y}, y \frac{\partial f}{\partial x}+\frac{\partial f}{\partial y} .
\end{aligned}
$$

These transformations, twice extended, have the forms

$$
\begin{array}{r}
z \frac{\partial f}{\partial x}-p^{2} \frac{\partial f}{\partial p}-p q \frac{\partial f}{\partial q}-3 p r \frac{\partial f}{\partial r}-(2 p s+q r) \frac{\partial f}{\partial s}-(p t+2 q s) \frac{\partial f}{\partial t}=0 \\
z \frac{\partial f}{\partial y}-p q \frac{\partial f}{\partial p}-q^{2} \frac{\partial f}{\partial q}-(2 p s+q r) \frac{\partial f}{\partial r}-(2 q s+p t) \frac{\partial f}{\partial s}-3 q t \frac{\partial f}{\partial t}=0 \\
y \frac{\partial f}{\partial x}+\frac{\partial f}{\partial y}+0 \frac{\partial f}{\partial z}+0 \frac{\partial f}{\partial p}-p \frac{\partial f}{\partial q}+0 \frac{\partial f}{\partial r}-r \frac{\partial f}{\partial s}-2 s \frac{\partial f}{\partial t}=0
\end{array}
$$

The solutions of the third equation are immediately

$$
z, p, r, y^{2}-2 x \equiv \rho_{1}, q+p y \equiv \rho_{2}, s+r y \equiv \rho_{3}, s^{2}-r t \equiv \rho_{4} .
$$

Introducing these as new variables, the first equation becomes

$$
0 \frac{\partial f}{\partial z}+p^{2} \frac{\partial f}{\partial p}+3 p r \frac{\partial f}{\partial r}+2 z \frac{\partial f}{\partial \rho_{1}}+p \rho_{2} \frac{\partial f}{\partial \rho_{2}}+\left(2 p \rho_{3}+r \rho_{2}\right) \frac{\partial f}{\partial \rho_{3}}+4 p \rho_{1} \frac{\partial f}{\partial \rho_{4}}=0
$$

of which the integrals are, in the original variables,

$z, \frac{p^{3}}{r} \equiv u, \frac{y^{2}-2 x}{2 z}+\frac{1}{p} \equiv v, \frac{q+p y}{p^{\prime}} \equiv w, \frac{s^{2}-r t}{p^{4}} \equiv m, \frac{s p-q r}{p^{3}} \equiv n$.

Introducing these as new variables into the second equation, it becomes

$$
0 \frac{\partial f}{\partial z}+2 u^{2} n \frac{\partial f}{\partial u}+w \frac{\partial f}{\partial v}+z \frac{\partial f}{\partial w}+0 \frac{\partial f}{\partial m}-\left(u n^{2}+u m\right) \frac{\partial f}{\partial n}=0 .
$$

The solutions are $z, m, w^{2}-2 v z, u n^{2}+u m, w\left(u n^{2}+u m\right)+z n$, or in the original variables : 
THE $G_{r}(r<5)$ OF $n$-DIMENSIONAL SPACE.

47

$$
\begin{gathered}
z, \frac{s^{2}-r t}{p^{4}}, \frac{(q+p y)^{2}}{p^{2}}-2 z\left(\frac{y^{2}-2 x}{2 z}+\frac{1}{p}\right), \frac{p^{3}}{r}\left[\left(\frac{p s-q r}{p^{3}}\right)^{2}+\frac{s^{2}-r t}{p^{4}}\right], \\
\frac{p^{2}(q+p y)}{r}\left\{\left(\frac{s p-q r}{p^{3}}\right)^{2}+\frac{s^{2}-r t}{p^{4}}\right\}+z\left(\frac{s p-q r}{p^{3}}\right) .
\end{gathered}
$$

In the usual way, the differential parameters are found to be

$$
\frac{p \phi_{y}-q \phi_{x}}{p}, \frac{p z \phi_{x}+(q+p y)\left(p \phi_{x}-q \phi_{y}\right)}{p^{2}} .
$$

(3) We will next consider the groups :

$$
\frac{\partial f}{\partial x}, x \frac{\partial f}{\partial x}+y \frac{\partial f}{\partial y}, x^{2} \frac{\partial f}{\partial x}+2 x y \frac{\partial f}{\partial y}
$$

(ii) $\frac{\partial f}{\partial x}, x \frac{\partial f}{\partial x}+y \frac{\partial f}{\partial y}, x^{2} \frac{\partial f}{\partial x}+\left(2 x y+y^{2}\right) \frac{\partial f}{\partial y}$;

(iii) $\frac{\partial f}{\partial x}, x \frac{\partial f}{\partial x}+y \frac{\partial f}{\partial y}, x^{2} \frac{\partial f}{\partial x}+2 x y \frac{\partial f}{\partial y}+y \frac{\partial f}{\partial z}$.

The first two transformations of these three groups are the same, and the partial differential invariants are easily found to be :

$$
z, \quad y p \equiv \rho_{1}, \quad y q \equiv \rho_{2}, \quad y^{2} r \equiv \rho_{3}, \quad y^{2} s \equiv \rho_{4}, \quad y^{2} t \equiv \rho_{5} .
$$

(i) Introducing these into the twice-extended form of the third transformation, it becomes

$$
0 \frac{\partial f}{\partial z}-2 \rho_{2} \frac{\partial f}{\partial \rho_{1}}+0 \frac{\partial f}{\partial \rho_{2}}-2\left(\rho_{1}+2 \rho_{1}\right) \frac{\partial f}{\partial \rho_{3}}-2\left(\rho_{2}+\rho_{5}\right) \frac{\partial f}{\partial \rho_{4}}+0 \frac{\partial f}{\partial \rho_{5}}=0 ;
$$

the solutions of which are

$$
\begin{aligned}
\approx, \rho_{2}, \rho_{5}, \rho_{1} \rho_{2}+\rho_{1} \rho_{5}-\rho_{4} \rho_{2},\left(\rho_{2}+\rho_{5}\right)^{2} \rho_{3} & -\frac{3}{2} \rho_{4}^{2} \rho_{2}-\rho_{4}^{2} \rho_{5} \\
& -\rho_{4}\left(\rho_{1} \rho_{2}+\rho_{1} \rho_{5}-\rho_{4} \rho_{2}\right) .
\end{aligned}
$$

Expressed in the original variables, these are :

$$
\begin{aligned}
& z, y q, y^{2} t, y^{2}(p q+p t y-q 8 y), y^{4}(q+t y)^{2} r-\frac{1}{2} y^{5} q s-y^{6} s^{2} t \\
& -y^{4} s(p q+p t y) \text {. }
\end{aligned}
$$


The differential parameters for this group are:

$$
y \phi_{y}, \quad y^{2} q \phi_{x}-y^{2} p \phi_{y} .
$$

(ii) By the new variables, the third member of the second group is

$0 \frac{\partial f}{\partial z}+\left(\rho_{1}-2 \rho_{2}\right) \frac{\partial f}{\partial \rho_{1}}-\rho_{2} \frac{\partial f}{\partial \rho_{2}}+\left(2 \rho_{3}-2 \rho_{1}-4 \rho_{4}\right) \frac{\partial f}{\partial \rho_{3}}$

$$
-2\left(\rho_{2}+\rho_{5}\right) \frac{\partial f}{\partial \rho_{4}}-2\left(\rho_{2}+\rho_{5}\right) \frac{\partial f}{\partial \rho_{5}}=0 \text {. }
$$

The solutions are :

$z, \rho_{1}-\rho_{5}, \rho_{1} \rho_{2}-\rho_{2}^{2}, \frac{\rho_{5}+2 \rho_{2}}{\rho_{2}^{2}}, \rho_{2}^{2} \cdot \rho_{3}-2 \rho_{2}^{2}\left(\rho_{4}-\rho_{5}\right)-\frac{3}{2} \rho_{2}^{4}\left(\frac{\rho_{5}}{\rho_{2}^{2}}+\frac{2}{\rho_{2}}\right)-4 \rho_{2}^{3}$. These can easily be expressed in the old variables. The differential parameters are found to be

$$
\frac{\phi_{y}}{q}, \quad y^{2} q\left(\phi_{x}-\phi_{y}\right)
$$

(iii) In the same way, the invariants for the third of the above groups are:

$$
\begin{array}{r}
z-y q, y^{2} t, y p+y^{2} q^{2}, y^{2} s+y^{2} q^{2}+2 y^{3} t \cdot q, \\
y^{2} r+2 y^{2} p q+4 y^{3} q s+4 y^{2} q^{2}+4 y^{4} q^{2} t,
\end{array}
$$

and the differential parameters are:

$$
y \phi_{y}, y \phi_{x}+2 z y \phi_{y} \text {. }
$$

(4) As the finding of the differential invariants of the remaining $G_{3}$ 's offers no special difficulties, we will merely state the results. We use $I_{1}, J_{1}$, for the invariants of the first order, and $I_{2}, I_{2}, K_{2}$, for those of the second order. The differential parameters can be easily computed for each group.

$$
\begin{aligned}
& \text { Group. } \\
& \text { (i) } \frac{\partial f}{\partial x}, \frac{\partial f}{\partial y}, \frac{\partial f}{\partial z}: I_{1} \equiv p ; \quad J_{1} \equiv q ; I_{2} \equiv r ; \quad J_{2} \equiv s ; K_{2} \equiv t . \\
& \text { (ii) } \frac{\frac{\partial f}{\partial x}, \frac{\partial f}{\partial y}, z \frac{\partial f}{\partial x}: I_{1} \equiv \frac{p}{q} ; \quad I_{2} \equiv \frac{r}{p^{3}} ; \quad J_{2} \equiv \frac{p s-q r}{p^{3}} ;}{\quad \therefore K_{2} \equiv \frac{p^{2} t-2 p q s+q^{2} r}{p^{3}}}
\end{aligned}
$$


THE $G_{r}(r<5)$ OF $n$-DIMENSIONAL SPACE.

(iii) $\overline{\frac{\partial f}{\partial z}, x \frac{\partial f}{\partial z}, y \frac{\partial f}{\partial z}}: I_{2} \equiv r ; \quad J_{2} \equiv s ; \quad K_{2} \equiv t$.

(iv) $\overline{\frac{\partial f}{\partial z}, x \frac{\partial f}{\partial z}, F(x) \frac{\partial f}{\partial z}} \mid: I_{1} \equiv q ; \quad I_{2} \equiv s ; \quad J_{2} \equiv t$.

(v) $\frac{\partial f}{\partial x}, \frac{\partial f}{\partial y}, y \frac{\partial f}{\partial x}+\frac{\partial f}{\partial z}: I_{1} \equiv p ; \quad J_{1} \equiv z+\frac{q}{p} ; \quad I_{2} \equiv r ;$

$$
J_{2} \equiv z+\frac{s}{r} ; \quad K_{2} \equiv r z^{2}+2 s z+t .
$$

(vi) $\frac{\partial f}{\partial x}, \frac{\partial f}{\partial y}, y \frac{\partial f}{\partial x}: I_{1} \equiv p ; \quad I_{2} \equiv r ; \quad J_{2} \equiv q r-p s$;

$$
K_{2} \equiv q^{2} r+2 p^{2} t-2 p q s .
$$

(vii) $\frac{\partial f}{\partial x}, \frac{\partial f}{\partial y},(x \pm y) \frac{\partial f}{\partial x}+y \frac{\partial f}{\partial y}+\frac{\partial f}{\partial z}: I_{1}=z+\log p$;

$$
\begin{aligned}
& J_{1} \equiv \frac{q}{p}-\log p ; \quad I_{2} \equiv \frac{r}{p^{2}} ; \quad J_{2} \equiv \frac{s-r \log p}{p^{2}} ; \\
& K_{2} \equiv \frac{p t-p r[\log p]^{2}-2 s \log p}{p^{3}} .
\end{aligned}
$$

(viii) $\frac{\partial f}{\partial x}, \frac{\partial f}{\partial y},(x+y) \frac{\partial f}{\partial x}+y \frac{\partial f}{\partial y} \mid: I_{1}=\frac{q}{p}-\log p ; \quad I_{2} \equiv \frac{r}{p_{2}} ;$

$$
J_{2} \equiv \frac{s-r \log p}{p^{2}} ; \quad K_{2} \equiv \frac{p t-p r[\log p]^{2}-2 s \log p}{p^{3}} .
$$

(ix) $\overline{\frac{\partial f}{\partial z}, x \frac{\partial f}{\partial z}, \frac{\partial f}{\partial x}-z \frac{\partial f}{\partial z}}: I_{1} \equiv x+\log q ; \quad I_{2} \equiv x+\log r$;

$$
J_{2} \equiv x+\log s ; \quad K_{2} \equiv x+\log t .
$$

(x) $\frac{\partial f}{\partial x}, \frac{\partial f}{\partial y}, x \frac{\partial f}{\partial x}+c y \frac{\partial f}{\partial y}+\frac{\partial f}{\partial z}: I_{1} \equiv z+\log p ; \quad J_{1} \equiv \frac{p^{c}}{q} ;$

$$
I_{2} \equiv \frac{p^{2}}{r} ; \quad J_{2} \equiv \frac{p^{1+0}}{8} ; \quad K_{2} \equiv \frac{p^{20}}{t} .
$$

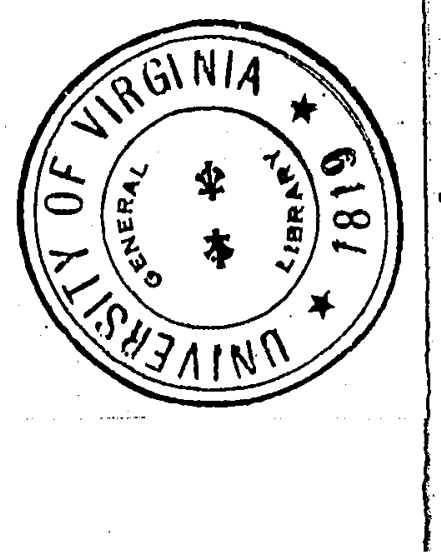


(xi)

$$
\begin{aligned}
& \frac{\partial f}{\partial x}, \quad \frac{\partial f}{\partial y}, x \frac{\partial f}{\partial x}+c y \frac{\partial f}{\partial y}: I_{1} \equiv \frac{p^{c}}{q} ; \quad I_{2} \equiv \frac{p^{2}}{r} ; \quad J_{2} \equiv \frac{p^{1+c}}{8} \\
& K_{2} \equiv \frac{p^{2 c}}{t} \text {. }
\end{aligned}
$$

(xii) $\frac{\partial f}{\partial z}, x \frac{\partial f}{\partial z},(1-c) x \frac{\partial f}{\partial x}+z \frac{\partial f}{\partial z}: I_{1} \equiv \frac{q^{1-0}}{x} ; \quad I_{2} \equiv \frac{q^{2 c=1}}{r}$;

$$
J_{2} \equiv \frac{q^{c}}{s} ; \quad K_{2} \equiv \frac{q}{t}
$$

(xiii) $\frac{\partial f}{\partial x}, x \frac{\partial f}{\partial x}, x^{2} \frac{\partial f}{\partial x}: I_{1} \equiv q ; \quad I_{2} \equiv \frac{s}{p} ; \quad J_{2} \equiv t$.

(xiv) $\frac{\partial f}{\partial x}, \frac{\partial f}{\partial y}, x \frac{\partial f}{\partial x}+z \frac{\partial f}{\partial y} ; I_{1} \equiv \log \frac{p}{q}+\frac{1}{q} ; \quad I_{2} \equiv \frac{t}{q}$;

and for this group, the remaining two invariants of the second order are found by quadratures from

$$
\frac{d s}{d q}=\frac{2 q+1}{q^{2}} s+b \epsilon^{a-1 / q}, \quad \frac{d r}{d q}=\frac{2+q}{q^{2}} r+\frac{2 s \epsilon^{a-1 / q}}{q},
$$

where for 8 in the second equation must be substituted its value in terms of $q$ from the first equation. In these two equations

$$
a \equiv \log \frac{p}{q}+\frac{1}{q}, \quad b=\frac{t}{q} .
$$

(xv) $\frac{\partial f}{\partial x}, \frac{\partial f}{\partial y}, x \frac{\partial f}{\partial x}: I_{1} \equiv q ; \quad I_{2} \equiv t ; \quad J_{2} \equiv \frac{p^{2}}{r} ; \quad K_{2} \equiv \frac{p}{s}$.

(xvi) $\frac{\partial f}{\partial z}, x \frac{\partial f}{\partial z}, z \frac{\partial f}{\partial z}: I_{2} \equiv \frac{r}{q} ; \quad J_{2} \equiv \frac{\varepsilon}{q} ; \quad K_{2} \equiv \frac{t}{q}$.

(xvii) $\frac{\partial f}{\partial z}, x \frac{\partial f}{\partial z}, \frac{\partial f}{\partial y}+z \frac{\partial f}{\partial z}: I_{1} \equiv \epsilon^{-y} q ; \quad I_{2} \equiv \frac{r}{q} ; \quad J_{2} \equiv \frac{s}{q} ;$

$$
\boldsymbol{K}_{2} \equiv \frac{t}{q}
$$


THE $G_{r}(r<5)$ OF n-DIMENgIONAL SPACE.

To these we will tabulate, for the sake of reference, the partial differential invariants obtained on pp. 44-48.

(xviii)

$\frac{\partial f}{\partial x}, \frac{\partial f}{\partial y}, z \frac{\partial f}{\partial x}+\eta(z) \frac{\partial f}{\partial y} ; I_{1} \equiv \frac{p}{q} ; \quad I_{2} \equiv \frac{q^{2} r-2 p q^{s}+p^{2} t}{q^{3}} ;$

$$
J_{2} \equiv \frac{q r-p s+(q s-p t) \eta^{\prime}(z)}{p q^{2}} ;[\text { see page 45]. }
$$

(xix)

$$
\begin{gathered}
\left.z \frac{\partial f^{\prime}}{\partial x}, z \frac{\partial f}{\partial y}, y \frac{\partial f}{\partial x}+\frac{\partial f}{\partial y}\right]: I_{1} \equiv\left(\frac{q+p y}{p}\right)^{2}-2 z\left(\frac{y^{2}-2 x}{2 z}+\frac{1}{p}\right) ; \\
I_{2} \equiv \frac{s^{2}-r t}{p^{4}} ; J_{2} \equiv \frac{p^{3}}{r}\left[\left(\frac{p s-q r}{p^{3}}\right)^{2}+\frac{s^{2}-r t}{p^{4}}\right] ; \\
K_{2} \equiv \frac{p^{2}}{r^{2}}(q+p y)\left[\left(\frac{p s-q r}{p^{3}}\right)^{3}+\frac{s^{2}-r t}{p^{4}}\right]+z\left(\frac{s p-q r}{p^{3}}\right) .
\end{gathered}
$$

$(\mathbf{x x})$

$$
\begin{aligned}
& \frac{\partial f}{\partial x}, x \frac{\partial f}{\partial x}+y \frac{\partial f}{\partial y}, x^{2} \frac{\partial f}{\partial x}+2 x y \frac{\partial f}{\partial y} \mid: I_{1} \equiv y q ; \quad I_{2} \equiv y^{2} t ; \\
& J_{2} \equiv y^{2}(p q+p t y-q s y) ; \quad K_{2} \equiv y^{4}(q+t y)^{2} r \\
& -\frac{1}{2} y^{5} q s-y^{6} s^{2} t-y^{4} s(p q+p t y) \text {. }
\end{aligned}
$$

(xxi) $\frac{\partial f}{\partial x}, x \frac{\partial f}{\partial x}+y \frac{\partial f}{\partial y}, x^{2} \frac{\partial f}{\partial x}+2 x y \frac{\partial f}{\partial y}+y \frac{\partial f}{\partial z}: I_{1} \equiv z-y q$;

$$
\begin{aligned}
& J_{1} \equiv y p+y^{2} q^{2} ; \quad I_{2} \equiv y^{2} t ; \quad J_{2} \equiv y^{2} s+y^{2} q^{2}+2 y^{3} t q \\
& K_{2} \equiv y^{2} r+2 y^{2} p q+4 y^{3} q^{s}+4 y^{2} q^{2}+4 y^{4} q^{2} t .
\end{aligned}
$$

(xxii) $\frac{\partial f}{\partial x}, x \frac{\partial f}{\partial x}+y \frac{\partial f}{\partial y}, x^{2} \frac{\partial f}{\partial x}+\left(2 x y+y^{2}\right) \frac{\partial f}{\partial y} \mid: I_{1} \equiv y^{2} p q-y^{2} q^{2}$;

$$
\begin{aligned}
& I_{2} \equiv y^{2}(s-t) ; \quad J_{2} \equiv \frac{t}{q^{2}}+\frac{2}{y q} ; \\
& K_{2} \equiv y^{4} q^{2} r-2 y^{4} q^{2}(s-t)-\frac{3}{2} y^{4} q^{4}\left[\frac{t}{q^{2}}+\frac{2}{y q}\right]-4 y^{3} q^{2}
\end{aligned}
$$




\section{CHAPTER III. \\ Observations and Applications.}

Many interesting investigations are suggested by the knowledge of the groups which have been found. We shall conclude this paper with a few remarks that have an important bearing on the general. problem of integration.

The Operations Necessary. for Reducing the $G_{r}(r<4)$ to Normal Forms.

1. As is well known if the $G_{1}$

$$
U_{1} f \equiv \xi_{1} p+\eta_{1} q+\zeta_{1} r
$$

is given, it can be' thrown into the form of a translation, $\bar{p}$, only by the integration of the simultaneous system

$$
\frac{d x}{\xi_{1}}=\frac{d y}{\eta_{1}}=\frac{d z}{\zeta_{1}}
$$

unless the path-curves of $U_{1} f$ are known.

2. If a given $G_{2}, U_{1} f, U_{2} f$, belongs to the type $p, x p$, it is easily seen that the reduction to normal form requires, in general, the integration of the simultaneous system (1). If the $G_{2}$ belongs to either of the other three types, the reduction can be accomplished by the integration of an ordinary differential equation of first order in two variables, together with certain quadratures.

3. Let us next consider the $G_{3}^{\prime}$ s.

I. When no linear relation exists.

If the $G_{3}$ belongs to the type ( $[4]$, table of $G_{3}$, page 41),

$$
p_{1}, x_{1} p_{1}+y_{1} q_{1}, x_{1}^{2} p_{1}+2 x_{1} y_{1} q_{1}+y_{1} r_{1},
$$

the reduction is accomplished as follows :

If the three transformations are given in the form

$$
U_{i} f \equiv \underset{52}{\xi_{i} p+\eta_{i} q+\zeta_{i} r} \quad(i=1,2,3)
$$


THE $G_{r}(r<5)$ OF $n$-DIMENSIONAL SPACE.

we know that there must exist three functions of $x, y, z-x_{1}(x, y, z)$, $y_{1}(x, y, z), z_{1}(x, y, z)$ - such that

$$
\begin{aligned}
& U_{1} f \equiv \xi_{1} p+\eta_{1} q+\zeta_{1} r=p_{1}, \\
& U_{2} f \equiv \xi_{2} p+\eta_{2} q+\zeta_{2} r=x_{1} p_{1}+y_{1} q_{1}, \\
& U_{3} f \equiv \xi_{3} p+\eta_{3} q+\zeta_{3} r=x_{1}^{2} p_{1}+2 x_{1} y_{1} q_{1}+y_{1} r_{1} .
\end{aligned}
$$

Hence $x_{1}$ is determined by

$$
U_{1}\left(x_{1}\right) \equiv \xi_{1} \frac{\partial x_{1}}{\partial x}+\eta_{1} \frac{\partial x_{1}}{\partial y}+\zeta_{1} \frac{\partial x_{1}}{\partial z}=1,
$$

$$
\begin{aligned}
& U_{2}\left(x_{1}\right) \equiv \xi_{2} \frac{\partial x_{1}}{\partial x}+\eta_{2} \frac{\partial x_{1}}{\partial y}+\zeta_{2} \frac{\partial x_{1}}{\partial z}=x_{1}, \\
& U_{3}\left(x_{1}\right) \equiv \xi_{3} \frac{\partial x_{1}}{\partial x}+\eta_{3} \frac{\partial x_{1}}{\partial y}+\zeta_{3} \frac{\partial x_{1}}{\partial z}=x_{1}^{2} .
\end{aligned}
$$

By DuBois-Raymond's method (see Lie's "Differentialgleichungen," page 553 ), $x_{1}$ can be determined from (2) by the integration of a Riccati equation.

Now considering that $x_{1}$ has been found and introduced as a new variable in place of $x, y_{1}$ is given by

$$
\begin{aligned}
& U_{1}\left(y_{1}\right) \equiv \frac{\partial y_{1}}{\partial x_{1}}+\eta_{1} \frac{\partial y_{1}}{\partial y}+\zeta_{1} \frac{\partial y_{1}}{\partial z}=0 \\
& U_{2}\left(y_{1}\right) \equiv x_{1} \frac{\partial y_{1}}{\partial x_{1}}+\eta_{2} \frac{\partial y_{1}}{\partial y}+\zeta_{2} \frac{\partial y_{1}}{\partial z}=y_{1} \\
& U_{3}\left(y_{1}\right) \equiv x_{1}^{2} \frac{\partial y_{1}}{\partial x_{1}}+\eta_{3} \frac{\partial y_{1}}{\partial y}+\zeta_{3} \frac{\partial y_{1}}{\partial z^{\prime}}=2 x_{1} y_{1} .
\end{aligned}
$$

Here $x_{1} \equiv x_{1}(x, y, z)$; and the third equation is not the same as the second. Dividing the third equation by the known function $x_{1}$, and solving for the partial derivatives, we get

$$
\frac{\partial y_{1}}{\partial x_{1}}=\rho_{1}+\sigma_{1} y_{1}, \quad \frac{\partial y_{1}}{\partial y}=\rho_{2}+\sigma_{2} y_{1}, \quad \frac{\partial y_{1}}{\partial z}=\rho_{3}+\sigma_{3} y_{1} .
$$

Hence $y_{1}$ is found by quadrature.

In the same way $z_{1}$ is given by

$$
U_{1}\left(z_{1}\right) \equiv \frac{\partial z_{1}}{\partial x_{1}}+\eta_{1} \frac{\partial z_{1}}{\partial y}+\zeta_{1} \frac{\partial z_{1}}{\partial z}=0
$$


WILLIAM B. STONE.

$$
\begin{aligned}
& U_{2}\left(z_{1}\right) \equiv x_{1} \frac{\partial z_{1}}{\partial x_{1}}+\eta_{2} \frac{\partial z_{1}}{\partial y}+\zeta_{2} \frac{\partial z_{1}}{\partial z}=0, \\
& U_{3}\left(z_{1}\right) \equiv x_{1}^{2} \frac{\partial z_{1}}{\partial x_{1}}+\eta_{3} \frac{\partial z_{1}}{\partial y}+\zeta_{3} \frac{\partial z_{1}}{\partial z}=y_{1},
\end{aligned}
$$

where $y_{1}$ is a known function. Hence the partial derivatives of $z_{1}$ are known, and $z_{1}$ is given by a quadrature.

In this case, therefore, the integration of a Riccati equation and certain quadratures are demanded. If the $G_{3}$ belongs to any one of the other four types $([10],[13],[17],[21]$, pages $42-43)$ of this class, the reduction is seen to require only quadratures.

II. When one linear relation exists.

If the $G_{3}$ belongs to one of the types ([8], [15], [19], [20], pages $41-43$ ), the reduction to normal form requires only quadratures. In the other cases of this class, the integration of an ordinary differential equation of first order in two variables is necessary.

III. When two linear relations exist.

If the $G_{3}$ belongs to the type [1], the reduction can be accomplished only by integrating a simultaneous system

$$
\frac{d x}{\xi_{1}}=\frac{d y}{\eta_{1}}=\frac{d z}{\zeta_{1}}
$$

If the $G_{3}$ belongs to either one of the other two types of this class $([5],[18])$, the reduction requires in the most unfavorable case the integration of an ordinary differential equation of the first order in two variables.

In the Leipziger Berichte, Vol. 47, pp. 494-499, Lie shows that if with the transformations of the $G_{3}$ of rotations

$$
\begin{aligned}
& X_{1} f \equiv y p-x q \\
& X_{2} f \equiv z q-y r \\
& X_{3} f \equiv x r-z p
\end{aligned}
$$

we form the functions $X_{3} \phi\left(X_{1} / X_{3}, X_{2} / X_{3}\right)$ where $\phi$ is arbitrary, these functions can be considered to be the characteristic functions of contact transformations. From this, it follows that this $G_{3}$ is a finite 
THE $G_{r}(r<5)$ OF n-DIMENSIONAL SPAOE.

sub-group of an infinite group of contact transformations, and several interesting properties are obtained from this fact. As we now have all the $G_{3}$ 's, we can use each one as the starting-point for theories similar to those deduced by $\mathrm{Lie}$ in the case of rotations.

In his "Berührungstransformationen" [Kap. 13], Lie has discussed the general problem of the integration of a non-linear partial differential equation of the first order, which is invariant under two transformations. As we can obtain a $G_{2}$, under which the equation is invariant when two transformations are known under which it is invariant, we can obtain Lie's results in an exceedingly simple manner, by using the typical forms of the $G_{2}^{\prime}$ 's, established above.

I. The Partial Differential Equation admits of a $G_{1}$. In this case, by the introduction of new variables, the transformation can be brought to the form of a translation $\partial f / \partial x$. Hence this shows that, in the new variables, the equation will be free of $x$ and will have the form

as is well known.

$$
F(y, z, p, q)=0
$$

II. The equation admits of a $G_{2}$.

(1) If the path-curves are the same, then by Theorem 5, p. 615, Lie's "Berührungstransformationen," the equation is linear in $p, q$. We see the same here for the two $G_{2}$ 's, which have the same pathcurves.

$$
\frac{\partial f}{\partial x}, z \frac{\partial f}{\partial x}
$$

By page 44, the typical invariant equation is

This is equivalent to

$$
F\left(y, z, \frac{p}{q}\right)=0
$$

$$
\alpha(y, z) \frac{\partial z}{\partial x}+\beta(y, z) \frac{\partial z}{\partial y}=0
$$

and since $z=$ const., is a solution, the problem can be solved com. pletely by a quadrature. 


$$
\frac{\partial f}{\partial x}, \quad x \frac{\partial f}{\partial x}
$$

By page 44, the typical invariant equation is

which is equivalent to

$$
F(y, z, q)=0 \text {, }
$$

$$
\frac{\partial z}{\partial y}=f(y, z), \quad \text { or } \quad \frac{d y}{1}=\frac{d z}{f(y, z)} \text {. }
$$

This is an ordinary differential equation of the first order, and thus far only, will the knowledge of the $G_{2}$ be of service in the integration in this case.

(2). The path-curves are different.

(i)

$$
\frac{\partial f}{\partial x}, \frac{\partial f}{\partial y}
$$

By page 44, the typical invariant equation is

$$
F(z, p, q)=0 \text {. }
$$

This equation is immediately integrable by quadrature.

$$
\frac{\partial f}{\partial z}, x \frac{\partial f}{\partial x}+y \frac{\partial f}{\partial y}+z \frac{\partial f}{\partial z}
$$

The invariant equation is

$$
F\left(\frac{x}{y}, p, q\right)=0 \text {. }
$$

The solution of this equation demands at most the integration of an ordinary differential equation of the first order.

III. Suppose the equation admits of a $G_{3}, U_{i} f,(i=1,2,3)$. In cases (i), (ii) under (1), pages 55-56, we have $U_{2}=\rho U_{1}$; so that the invariant partial differential equation of first order, $\Omega=0$, is linear and $\rho$ is one solution. Then if $U_{3}=\sigma \cdot U_{1}$, where $\sigma$ is not a func. tion of $\rho, \sigma$ is the other solution. $O r$ if no linear relation connects $U_{3}$ and $U_{1}$, the other solution of $\Omega=0$ can be found by quadrature. But if $\rho=F(\sigma)$, above, the integration of an ordinary differential equation of first order is still necessary to integrate $\Omega=0$. 
THE $G_{r}(r<5)$ OF $n$-DIMENSIONAL SPACE.

In case (2), (ii), Lie has shown that without reducing the $G_{2}, U_{1}$, $U_{2}$ to normal form, the integration of the invariant differential equation $\Omega(x, y, z, p, q)=0$ may be made to depend upon the integration of an integrable total equation

$$
P d x+Q d y+R d z=0 .
$$

Then if $P \xi_{3}+Q \cdot \eta_{3}+R \zeta_{3} \neq 0$, this equation is integrable by quadratures. [See Page's "Note on the Invariant Total Differential Equation $P d x+Q d y+R d z=0$," Annals of Mathematics, Vol. 12, No. 6.]

If the $G_{3}$ can be reduced to normal form without an integration, as is often possible, the invariant partial differential equation of first order must take the form (see pages 48-51],

or

$$
y q=\Omega(z)
$$

or

(iii)

$$
y q(y p=y q)=\mathbf{\Omega}(z) \quad \text { or } \quad y q=\text { const. }
$$

$$
y q-z=\text { const. }
$$

Each of these equations is integrable by quadrature.

If $I_{1}, J_{1}$, are the total differential invariants, first order, of any given $G_{3}$, the equation

$$
\Omega\left(I_{1}, J_{1}\right)=0
$$

will be an invariant Monge equation. Its integration depends, (see Lie, "Berührungstransformationen," page 553), upon that of the corresponding non-linear partial differential equation of the first order

$$
F(x, y, z, p, q)=0,
$$

which of course is also invariant and is handled by the method above.

It is interesting to note that $G_{3}{ }^{\prime}$ s belonging to the types $[1],[2]$, $[3],[5],[6],[7],[11],[14],[15],[16],[18],[19],[20]$, 
leave no Monge equation, but only integrable total equations of first order of the form

$$
P d x+Q d y+R d z=0
$$

invariant. This equation is integrable by quadrature if

$$
P \xi_{i}+Q \eta_{i}+R \zeta_{i} \neq 0
$$

for any value of $i=1,2,3$. These groups consequently leave no non-linear partial differential equation of the first order invariant. The other types may leave both Monge equations and equations of form (1) invariant.

From the partial differential invariants, we see that every $G_{3}$ in normal form leaves a partial differential equation of first order invariant except

$$
r, x r, y r
$$

In this, the invariant partial differential equation is of at least the second order.

We saw above that certain $G_{3}^{\prime}$ 's leave no Monge equations invariant, and hence no non-linear partial differential equations of first order. This was evident, $a$ priori, if any transformation of the $G_{3}$ was connected with any other of the $G_{3}$ by a linear relation, (see Lie, "Berührungstransformationen," Kap. 13). But the $G_{3}^{\prime}$ 's

and

$$
p, q, z p+\eta(z) q \quad(\eta \neq \mathrm{c} \cdot z)
$$

$$
p, x p+y q, x^{2} p+2 x y q
$$

are exceptional, as no such linear relation exists; and still only a linear partial differential equation of first order can be invariant under each of them.

By the method developed by Lie, (Leipziger Berichte, Oct. 10, 1893, pages 98, ff.), if a partial differential equation of order $m$, $\Omega^{(m)}=0$, is invariant under a known $G_{3}$, we can find, by integrating an ordinary differential equation of $m$ th order in two variables, 
THE $G_{r}(r<5)$ OF $n$-DIMENSTONaL SPAgE.

$\infty^{m+2}$ integral surfaces of $\Omega^{(m)}=0$, which are composed of path curves of the general transformation of the $G_{3}$,

$$
U f \equiv \sum_{i=1}^{3} a_{i} U_{i} f
$$

Hence if the form of $\Omega^{(m)}=0$ shows that it is invariant under a known $G_{3}$, it is frequently possible to find $\infty^{m+2}$ integral surfaces of $\Omega^{(m)}=0$ by Lie's method. Of course the operations are much simplified if the $G_{3}$ has been thrown into normal form, and the path curves of $U f$ have been determined.

It is easily seen that the most general transformation of space that leaves directions invariant belongs to one of the $G_{4}$ 's. The coordinates of a line element in space are $x, y, z, y_{1}, z_{1}$, and if the directions are to be invariant, the increments of $y_{1}$ and $z_{1}$ must be zero. Thus we have

$\eta_{1} \equiv \frac{d \eta}{d x}-y_{1} \frac{d \xi}{d x} \equiv \eta_{x}+\eta_{\nu} \cdot y_{1}+\eta_{s} \cdot z_{1}-y_{1}\left(\xi_{x}+\xi_{y} \cdot y_{1}+\xi_{z} \cdot z_{1}\right)=0$

$\zeta_{1} \equiv \frac{d \zeta}{d x}-z_{1} \frac{d \xi}{d x} \equiv \zeta_{x}+\zeta_{y} \cdot y_{1}+\zeta_{z} \cdot z_{1}-z_{1}\left(\xi_{x}+\xi_{y} \cdot y_{1}+\xi_{x} \cdot z_{1}\right)=0$

Since these relations are independent, we have

$$
\xi_{y}=\xi_{x}=\eta_{x}=\eta_{x}=\zeta_{x}=\zeta_{y}=0, \quad \xi_{x}=\eta_{y}=\zeta_{x} .
$$

Whence, immediately $\xi \equiv m x+\alpha, \eta \equiv m y+\beta, \zeta \equiv m z+\gamma ; m$, $\alpha, \beta, \gamma$, being arbitrary constants. Hence the transformation is

$$
(m x+\alpha) \frac{\partial f}{\partial x}+(m y+\beta) \frac{\partial f}{\partial y}+(m z+\gamma) \frac{\partial f}{\partial z} .
$$

This is recognized at once as the most general transformation of the fifth group under VI, (see table $G_{1}^{\prime}$ 's, p. 32), obtained by putting the arbitrary constants $a$ and $c$ equal to unity.

This $G_{4}$ is of importance in the problem of spherical representation of curves.

In the same way, it can be shown that the transformation obtained above is the most general one that leaves the direction of surface elements invariant. 
60

WILLIAM B. STONE.

We notice that there is only one $G_{2}$ in one variable; that there are four in two variables, and four in $n$-variables. There is one $G_{3}$ in one variable, eleven in two variables, twenty-one in three variables, and twenty-one in $n$-variables. There are $16 G$,'s in two variables, 71 in three variables, 105 in four variables, and 105 in $n$-variables. That for these three cases there is the same number of $G_{r}^{\prime}$ 's in $r$-varixales as in $n$-variables is to be expected according to Theorem 84 , page 458, of the first volume of Lie's "Theorie der Transformationsgruppen." It will readily be seen that more is shown in the abovegiven result than is stated in the theorem referred to. 


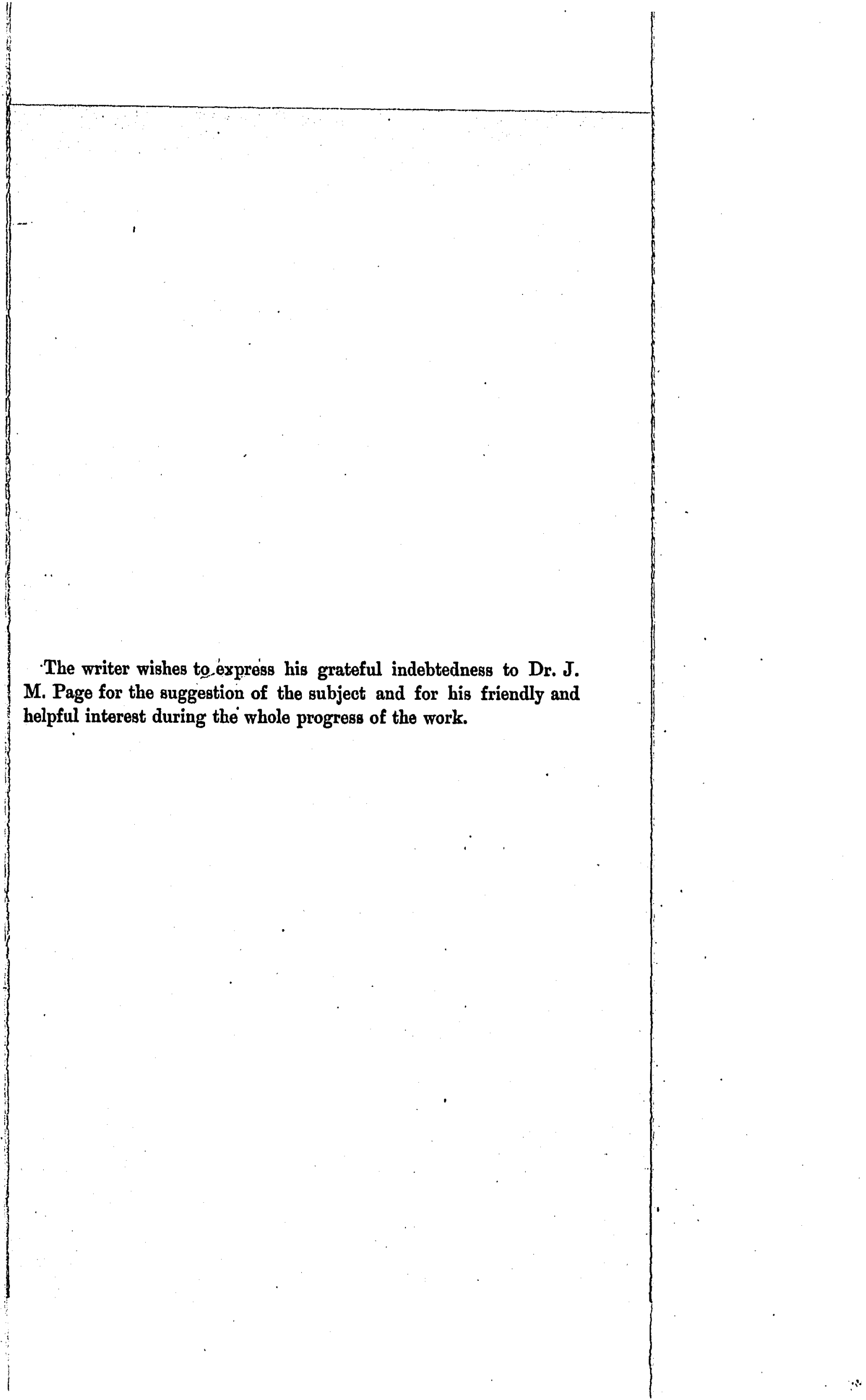

